\title{
Measurement of Birefringence for Optical Recording Disk Substrates
}

\author{
Hong Fu, S. Sugaya ${ }^{\dagger}$, J.K. Erwin, T. Goodman, Z. Yan, W.J. Tang and \\ M. Mansuripur \\ Optical Sciences Center \\ University of Arizona \\ Tucson, Arizona 85721
}

\begin{abstract}
The birefringence of bare and coated substrates for magneto-optical recording is experimentally investigated using ellipsometry at the wavelengths of $632.8 \mathrm{~nm}$ and $780 \mathrm{~nm}$. We measure the rotation and ellipticity of the polarization state of the reflected or transmitted light for different incident angles and different orientations of the incident linear polarization. The measured data is then fitted by a computer program which solves the Maxwell equations for the plane-wave propagation in a multilayer structure and minimizes the error between the measured and calculated data by adjusting the unknown parameters of the multilayer. This approach enables us to determine orientations of the three principal axes in the substrate and the corresponding refractive indices. A special feature of our ellipsometers is that a glass hemisphere is placed in contact with the substrate, which eliminates the refraction of the incident light and enables a maximum propagation angle of $70^{\circ}$ (with respect to the normal) in the substrate. This increases the sensitivity of the measurement. Certain anomalies have been observed, which we believe are associated with the presence of grooves on these substrates.
\end{abstract}

† Visiting scientist from Functional Devices Research Laboratories, NEC Corporation, Japan. 


\section{INTRODUCTION}

Birefringence is a phenomenon observed in optically anisotropic media in which the refractive indices depend on the polarization direction. The polarization-dependent phase-shifts due to birefringence of the plastic substrates of magneto-optical (MO) disks can easily degrade the performance of MO readout systems [1-7]. As the MO data storage systems progress towards high resolution and ultrafast speeds, the birefringence has become an increasingly important probilem.

Birefringence in plastic substrates is due to preferential molecular orientation and intemal stresses. The refractive index is higher when the electric field of the light beam is parallel to the molecular chain direction. For injection-molded polycarbonate substrate the fluid shear during the injection molding process acts to align the polycarbonate chains in the plane of the disk. Therefore, the refractive indices $n_{1}$ and $n_{2}$ for the in-plane electric field components $E_{1}$ and $E_{2}$ of light are much larger than $n_{3}$ for the perpendicular component $E_{3}$. The characterization of substrate birefringence has been the subject of several papers [6,8-11], which confirmed the above picture and found that $n_{1}-n_{3}$ is on the order of $10^{-4}$, while $\left|n_{1}-n_{2}\right|$ is on the order of $10^{-5}$. However, the previous work is limited in the following aspects: First, the formula $[8,11]$ by which the experimental data were processed applies only to transparent single-layer media under the assumption that the principal axes of the index ellipsoid are fixed in the radial, track and perpendicular directions. The phase-shifts due to the multilayers and due to the tilt of the index ellipsoid could not be taken into account. Second, due to refraction at the interface between air and substrate, the propagation angle of the light in the substrate is usually small. For 
example, $70^{\circ}$ incident angle in air corresponds to $36.5^{\circ}$ propagation angle in the polycarbonate substrate $(n=1.58)$. So the beam possesses only a small $E_{3}$ component and the experimental sensitivity to $n_{3}$ is limited.

The present paper describes measurements of the substrate birefringence using one ellipsometer with wavelength $\lambda=632.8 \mathrm{~nm}$ and another with $\lambda=780 \mathrm{~nm}$. We remove the first restriction of the previous work by using a computer program which solves the Maxwell equations for a plane-wave propagating in a multilayer structure including the substrate [12]. This approach enables us to determine the three principal axes of the substrate and the corresponding refractive indices. To overcome the second limitation, we have placed a hemispherical glass lens atop the substrate to eliminate refraction. A maximum propagation angle of $70^{\circ}$ in the substrate has thus been achieved. We measured the rotation angle and ellipticity of the polarization state of the reflected and/or transmitted light as functions of the incident angle for linearly polarized incident beam. The investigated substrate materials include glass, polycarbonate plastic (bare and coated), and amorphous polyolephin plastic (bare). Measurements were performed on regions with or without grooves. For the former case, the grooves were either filled with index-matching fluid or left empty. Different angles between the plane of incidence and the direction of grooves were used in the measurements. This paper is organized as follows. The two ellipsometer systems and measurement procedures are described in Sec. II, along with a description of the data analysis procedure using the multilayer program. The measurement results are described in Sec. III. Sec. IV contains some concluding remarks. 


\section{ELLIPSOMETER SYSTEMS AND THE MEASUREMENT METHOD}

\section{II.1 The VAE633 System}

Figure 1 shows the experimental setup. The light source is a HeNe laser with $\lambda=632.8 \mathrm{~nm}$. As the beam first passes through the polarizer and the quarter-wave plate (QWP), whose fast axis is at $45^{\circ}$ to the transmission axis of the polarizer, it becomes circularly polarized. The second polarizer selects the linear polarization direction of the incident beam. A hemispherical glass lens is placed in contact (using index-matching fluid) with the surface of the sample. This hemisphere is actually slightly less than one half of a sphere, and is mounted in such a way that the geometric center of the spherical surface is on the back side of the substrate. The light enters normally into the hemisphere, goes through the substrate, and is reflected from the back side of the substrate. After reflection, the beam passes through another QWP with an adjustable fast-axis orientation. The beam then goes through a Wollaston prism which can be rotated about the beam. Finally, the two beams of mutually perpendicular polarization are detected by the photodetectors.

The hemispherical lens eliminates refraction at the air-substrate interface, thus producing a larger propagation angle within the substrate. For example, in polycarbonate substrate with $n=1.58$, without the glass hemisphere one can reach a maximum angle of $39^{\circ}$ at grazing incidence (i.e. $\left.\theta_{\mathrm{inc}}=90^{\circ}\right)$, while with the hemisphere in place $\left(n_{\text {spbere }}=1.52\right)$, the propagation angle inside the substrates is $64^{\circ}$ at $\theta_{\text {inc }}=70^{\circ}$.

\section{II.2 The VAE780 System}


The setup is schematically shown in Fig. 2 (a). The operation principle is similar to the VAE633 system. One of the main differences is that all the moving parts are mounted in one arm, which can be rotated in both polar and azimuthal directions. The alignment of the system is thus easier to maintain. The freedom of two-dimensional rotation is particularly valuable to measure the effect of grooves, which are found to be sensitive to both polar incident angle $\theta_{\text {inc }}$ and the azimuthal incident angle $\Phi_{\operatorname{ixc}}$ relative to the radial direction. The linearly polarized light from the laser diode $(\lambda=780 \mathrm{~nm})$ passes through the beam splitter and enters normally through the hemisphere before it reaches the sample. The direction of incident polarization is controlled by rotating the laser in its socket. The hemisphere is used in different ways, depending on whether the sample is transparent or reflective. For transparent samples, two hemispheres are used, see Fig.2 (b). The one on the top is transparent and is used to eliminate refraction. The one at the bottom is coated with aluminum and reflects the beam normally so that the light goes back along the incident path. In this configuration the light passes twice through the substrate. For coated samples, we only need the top hemisphere, see Fig.2 (c). Half of this hemisphere is coated with aluminum. The beam enters from the uncoated side, gets reflected from the bottom of the sample, bounces off the coated side of the hemisphere, and retraces its path. Thus the beam passes four times through the substrate. In both transmission and reflection modes, the use of hemisphere(s) does not introduce phase shift or relative amplitude change between $p$ and $s$ components upon entering and exiting the substrate. Therefore, the measured values of rotation and ellipticity are solely determined by the properties of the sample. As the light beam returns, it is routed by the beam splitter into the detector channel, where its polarization state is determined by the use of the QWP, a Wollaston prism and two detectors. 


\section{II.3 The Measurement Principle}

The measured quantities are the rotation angle $\theta$ and ellipticity angle $\varepsilon$ of the reflected beam. The definitions of $\theta$ and $\varepsilon$ and the corresponding measurement procedures are described below. The p- and s-polarization directions for incident and reflected beams in VAE630 and VAE780 systems are defined in Fig. 1 and Fig.2 (a), respectively. The polarization angle $\psi_{\text {pol }}$ of the incident beam is measured from the p-direction, Fig. 3 (a), and can be read from the setting of the polarizer in VAE633 and the laser diode in VAE780. In general, due to phase retardation between the $\mathrm{p}$ - and s-components, the reflected beam is either left- or right-handed elliptically polarized (LEP or REP). Figure 3 (b) shows that the ellipse can be characterized by the orientation of its major axis, $\theta_{\text {axin }}$, and the ellipticity angle $\varepsilon$. By definition $|\tan \varepsilon|$ is the ratio of the minor to major axis, and $\varepsilon<0$ for LEP and $\varepsilon>0$ for REP. Thus, $-45^{\circ} \leq \varepsilon \leq 45^{\circ}$. In particular, $\varepsilon=-45^{\circ}$ (or $+45^{\circ}$ ) corresponds to LCP (or RCP), and $\varepsilon=0$ corresponds to linearly polarized light. When the fast-axis of the QWP is parallel to the major axis of the ellipse, the beam passing through it becomes linearly polarized. Its polarization angle $\theta_{\mathrm{w}}$ can be smaller or greater than $\theta_{\text {axis }}$, depending on whether the beam before the QWP is LEP or REP. These two cases are shown in Figs.3 (c) and (d). $\theta_{2 x i s}$ and $\theta_{w}$ can be measured simultaneously by adjusting the orientations of QWP and the Wollaston prism, until one of the detectors receives no light. Once extinction is achieved, the orientation of the fast-axis of the QWP gives $\theta_{\text {axis, }}$, while the Wollaston orientation gives $\theta_{\mathrm{w}}$. The rotation and ellipticity angles of the reflected beam are then given by:

$$
\theta=\theta_{\text {ario }}-\psi_{\text {pol }}, \varepsilon=\theta_{W}-\theta_{\text {acis }} \text {. }
$$

In practice, the error for the measured value of $\varepsilon$ is less than $1^{\circ}$, as estimated from repeated 
measurements. The error for $\theta$ depends on the value of $\varepsilon$. For $|\varepsilon|<40^{\circ}$, ertor of less than $1^{\circ}$ can be achieved. For $|\varepsilon|>40^{\circ}$, the ellipse is close to a circle and the orientation of the major axis is hard to measure. The repeatability of $\theta$ in such cases is within several degrees.

\section{I.4 Data Fitting with the Multilayer Program}

The first part of the multilayer program [12] solves the Maxwell equations for a plane wave obliquely incident upon a multilayer structure consisting of a given number of layers. Each layer is characterized by its dielectric tensor and thickness. With adequate restrictions on the tensor elements, different types of media (isotropic, birefringent, magneto-optic, etc.) can be described. Figure 4(a) shows the quadrilayer structure of a typical MO disk. This structure is used in data fitting for coated substrates. For bare substrates, we consider the substrate alone without additional layers. The hemisphere material $\left(\mathrm{n}_{\mathrm{aphere}}=1.52\right)$ and the index-matching fluid $\left(\mathrm{n}_{\mathrm{fuid}}=\right.$ 1.52) are treated as the medium of incidence. Since the optical paths in VAE633 and that in the transmission and reflection modes of VAE780 are different, three versions of the multilayer program were used in the data analysis. The second part of the program searches the best values of unknown parameters by minimizing the error, defined below, between the measured and the calculated data:

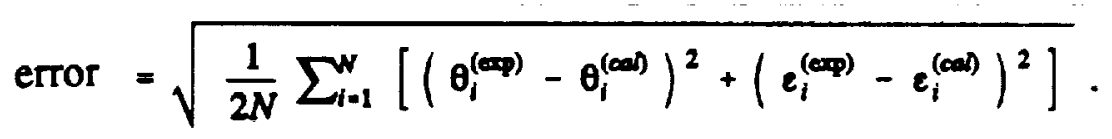

In this equation, the superscript "exp" stands for experimental data, and "cal" for calculated data, $\mathbf{N}$ is the total number of the measurements on a given sample. The unknown parameters are the refractive indices, the orientation angles of the principal axes of the ellipsoid of 
birefringence and the structure parameters of the multilayer. In our Convex computer system, it takes only a few minutes to search for 10 unknown parameters in fitting 120 data points.

In Fig.4 (a) $\mathrm{X}$ and $\mathrm{Y}$ denote the in-plane radial and track directions of an optical disk, and $\mathrm{Z}$ the normal direction. In addition to the incident angle $\theta_{\text {inc }}$, we use $\Phi_{\text {inc }}$ to specify the azimuth of the incident $\mathrm{k}$-vector. For all the samples, we found that one principal axis (3) is always along the perpendicular direction, but the in-plane axes ( 1 and 2) may be rotated in the $X-Y$ plane, see Fig. 4 (b). To be specific, we let 1 be the in-plane axis with the larger refractive index, and use $\psi_{\mathrm{im}-\mathrm{planc}}$ to denote the in-plane rotation of axis 1 from the radial direction.

There are two aspects of the actual systems that are not taken into account in the multilayer program. One is that the beam is not a plane wave, but a focused beam with $0.5 \mathrm{~mm}$ diameter that converges to a $50 \mu \mathrm{m}$ spot at the bottom of the substrate. For thick samples, the two reflected beams from the front and back surfaces are spatially separate and do not interfere with each other, while in the program the two beams overlap. However, since the reflection at the interface between the hemisphere and the substrate is weak, the interference only causes small fluctuation in the calculated curves. Therefore, the plane wave approximation is acceptable. Another aspect is the structure of the grooves on the surface of the substrate, which are usually $0.5 \mu \mathrm{m}$ wide, $70 \mathrm{~nm}$ deep, and have a periodicity of $1.6 \mu \mathrm{m}$. The effect of the grooves on polarized light has not been fully investigated. We found that, for bare substrates, the data measured at $\Phi_{\mathrm{inc}}=0^{\circ}$ can be matched very well, while for coated substrates, good match can be obtained for $\Phi_{\mathrm{inc}}=90^{\circ}$. For other values of $\Phi_{\mathrm{inc}}$, there is usually mismatch between the 
measured data and theory. In these occasions we are probably witnessing anomalous behavior caused by the grooves.

\section{RESULTS}

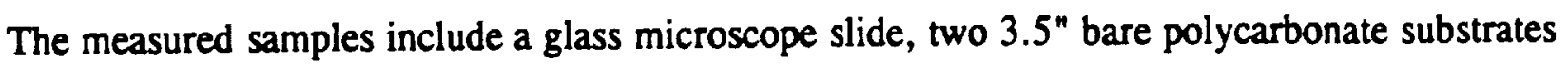
(PC3.5B01 ${ }^{1}$ and $\mathrm{PC} 3.5 \mathrm{B02}$ ), two 5.25" polycarbonate bare substrates (PC5.25B01 and PC5.25B02), one 5.25" amorphous polyolephin substrate (APO5.25B01), two 3.5" MO disks (PC3.5C01 and PC3.5C02), three 5.25" MO disks (PC5.25C01, PC5.25C02, PC5.25C03). All coated disk substrates are polycarbonate plastic. The main results of these measurements are summarized in Table 1.

\section{III.1 The Glass Sample}

To check the VAE633 system, we measured a glass microscope slide with thickness $d=1.2$ $\mathrm{mm}$. Glass is believed to be isotropic with a refractive index close to that of the hemisphere and index-matching fluid. So the optical path is simple (e.g. no multiple reflections) and the problem should be well described by the well-known formulas for reflectivity at a single interface $[13,14]$.

Fig. 5 shows $\theta$ and $\varepsilon$ as functions of the incident angle in the range $10^{\circ} \leq \theta_{\text {inc }} \leq 70^{\circ}$ for $\psi_{\text {pol }}$ $=45^{\circ}$. The measured data in (a) and (b) are same. The calculated curves in (a) are obtained by

${ }^{1}$ In this notation PC stands for polycarbonate (APO stands for amorphous polyolephin), the number 3.5 for the disk diameter in inches, $B$ for bare substrate ( $C$ for coated substrate), and 01 is the serial number. 
assuming that the glass is isotropic. The match gives $n_{g \text { lass }}=1.54$. We see that the rotation angles can be matched very well, but the non-zero ellipticity for $\theta_{\text {inc }} \leq 41^{\circ}$ (critical angle of total internal reflection) cannot be matched. The average fitting error is $1.9^{\circ}$. To obtain a better match, we allowed the glass to have a small amount of birefringence. The curves in Fig. 5(b) show the best match with an average error of $0.8^{\circ}$. The fitted refractive indices are $n_{1}=1.54$, $\left(n_{1}-n_{2}\right)=6 \times 10^{-6},\left(n_{1}-n_{3}\right)=-1 \times 10^{-6}$ and $\psi_{\text {in-plane }}=-9.2^{\circ}$. In this particular case where the sample has no grooves, $\mathrm{X}$ and $\mathrm{Y}$ stand for in-plane directions along the two rectangular sides of the microscope slide.

The basic features of the plots of $\theta$ and $\varepsilon$ versus $\theta_{\mathrm{inc}}$ can be understood using the reflectivities for $E_{p}$ and $E_{s}$ for dielectric interface $[13,14]$. Below the critical angle for the total internal reflection $\left(\theta_{i n c} \leq 41^{\circ}\right)$, the reflectivities are real and non-equal, i.e. the values of $\left|E_{p}\right|$ and $\left|E_{\mathrm{a}}\right|$ will change after reflection but the phase difference should remain zero. Therefore, the reflected beam is still linearly polarized $(\varepsilon=0)$ but in a different direction $(\theta \neq 0)$. The measured non-zero $\varepsilon$ indicates that there exists a small amount of birefringence. At the critical angle the behavior of $\theta$ and $\varepsilon$ changes drastically. We mention in passing that the total reflection occurs at the interface between the glass sample and air, but the critical incident angle is determined by the refractive index of the hemisphere, as can be readily seen from Snell's law. In VAE 633 , we found that the critical angle is $41.3^{\circ}$, which is in agreement with $n_{\text {sphere }}=1.52$. Above $\theta_{i \mathrm{ic}}=42^{\circ}$, both $E_{p}$ and $E_{s}$ are totally reflected and acquire different phase shift, i.e. $\left|E_{\mathrm{p}}\right|=\left|\mathrm{E}_{\mathrm{a}}\right|, \phi_{\mathrm{p}} \neq \phi_{\mathrm{s}}$. So we have $\varepsilon \neq 0$. 


\section{III.2 Sample PC3.5B01 and PC3.5B02}

The measured point is located at about $3 \mathrm{~cm}$ from the center of the disk and at the center of a sector with grooves. To reduce diffraction effects, the groove side of the substrate was placed in contact to the hemisphere and the grooves were thus filled with index matching fluid. The inplane projection of the plane of incidence is perpendicular to the track direction $\left(\Phi_{\mathrm{inc}}=0^{\circ}\right)$.

Figures 7 (a) and (b) show the measurement results and the matched curves for sample PC3.5B01 with $\psi_{\text {pol }}=30^{\circ}$ and $60^{\circ}$, respectively. The best fit for the data set with $\psi_{\text {pol }}=30^{\circ}$ gives $n_{1}=1.58,\left(n_{1}-n_{2}\right)=1.5 \times 10^{-5},\left(n_{1}-n_{3}\right)=5.87 \times 10^{-4}$ and $\psi_{\text {m-pleace }}=-0.3^{\circ}$. The fitting error is $2.9^{\circ}$. The parameters found for $\psi_{\text {pot }}=60^{\circ}$ are: $n_{1}=1.58,\left(n_{1}-n_{2}\right)=1.3 \times 10^{-5},\left(n_{1}-n_{3}\right)$ $=5.87 \times 10^{-4}$, and $\psi_{\text {in-plese }}=-5.3^{\circ}$. The average error for $\psi_{\text {pol }}=60^{\circ}$ is $1.7^{\circ}$, smaller than that for $\psi_{\text {pol }}=30^{\circ}$. We see that the two sets of fitted parameters are nearly identical. Ingnoring the small values of $\psi_{\text {in-plane }}$, one can assert that the principal axes are in fact along the radial, track and perpendicular directions.

Some of the features shown in Fig.6 are common to all the substrates and can be easily explained. First, the absolute values of $\theta$ and $\varepsilon$ are small at small $\theta_{\text {ixc }}$. The reason is that the beam close to normal incidence only experiences the small in-plane birefringence, but not the larger vertical birefringence. The second feature is that the oscillation interval, i.e. the difference between incident angles corresponding to two adjacent peaks, decreases with increasing $\theta_{\text {inc }}$. For example, the peaks of $\varepsilon$ in (a) appear at $\theta_{\text {icc }}=22^{\circ}, 37^{\circ}, 49^{\circ}, 60^{\circ}$ and $68^{\circ}$. The corresponding intervals are $15^{\circ}, 12^{\circ}, 11^{\circ}$ and $8^{\circ}$, and decrease monotonically. This feature 
could be understood as the change of optical path length per incident angle increases with $\theta_{\mathrm{inc}}$. Another feature is that $\theta$ always shows a $90^{\circ}$ jump when $\varepsilon=45^{\circ}$, see the data points at $\theta_{\text {inc }}=$ $22^{\circ}$ in (a). What happens here is that the polarization ellipse is very close to a circle. A slight decrease in the major-axis or increase in the minor-axis causes exchange of the axes and lead to a $90^{\circ}$ jump. This transition is shown in Fig. 7 .

Sample PC3.5B02 is another substrate in the same series of product as PC3.5B01. We measured the same position ( $3 \mathrm{~cm}$ from the center of the disk and center of a sector with grooves) in both VAE633 and VAE780 for $\Phi_{\text {inc }}=0^{\circ}$ and $\psi_{\text {pol }}=60^{\circ}$. The data taken with VAE633 has a shape similar to that shown in Fig.6 (b). However, the best fit gives $\psi_{\text {in-pine }}=-50.3^{\circ}$. In comparison with PC3.5B01, the in-plane birefringence is three times smaller: $\left(n_{1}-n_{2}\right)=0.5 \times 10^{-5}$ and vertical birefringence is somewhat larger $\left(n_{1}-n_{3}\right)=6.26 \times 10^{-4}$. PC3.5B02 was also measured with VAE780 in the transmission mode. Figure 8 shows the measured data and the best fit. The best match gives $\psi_{\text {in-plane }}=56.7^{\circ}$. The fitted parameters are: $n_{1}=1.58,\left(n_{1}-n_{2}\right)=1.9 \times 10^{-5}$, and $\left(n_{1}-n_{3}\right)=5.83 \times 10^{-4}$.

\section{III.3 Sample APO5.25B01}

The measured position is located $5 \mathrm{~cm}$ from the center of the disk and in the middle of a sector with grooves. The groove side is in contact with the hemisphere. The sample was measured with VAE633 at $\Phi_{\mathrm{icc}}=0^{\circ}$ for $\psi_{\mathrm{pol}}=60^{\circ}$. The measured data and the best fit are shown in Fig. 9 . Both in-plane and vertical birefringence are small: $n_{1}=1.50,\left(n_{1}-n_{2}\right)=0.4 \times 10^{-5},\left(n_{1}-n_{3}\right)=$ $1.3 \times 10^{-5}$ and $\psi_{\text {in-plane }}=-72.5^{\circ}$. 


\section{III.4 Sample PC5.25B01 and PC5.25B02}

These two samples are from the same series of product. The measured point on smaple PC5.25B01 was located $5 \mathrm{~cm}$ from the disk center and in the middle of a sector with grooves. The groove side was faced to the hemisphere and filled with index matching fluid. The measurement was performed with $\Phi_{\text {inc }}=0^{\circ}$ and $\psi_{\text {pol }}=60^{\circ}$ with VAE780. The measured data and the match are shown in Fig. 10. The fitted parameters are: $n_{1}=1.58,\left(n_{1}-n_{2}\right)=1.3 \times 10^{-5}$, $\left(n_{1}-n_{3}\right)=6.22 \times 10^{-4}$ and $\psi_{\text {in-plane }}=66^{\circ}$.

For Sample PC5.25B02 we measured the region without grooves: a point located $2.5 \mathrm{~cm}$ from the center of the disk with VAE633 for $\psi_{\text {pol }}=30^{\circ}, 45^{\circ}, 60^{\circ}$ and $\Phi_{\text {pc }}=90^{\circ}$. Figure 11 (a)-(c) show the measured data and the fits. No match has been found for $\theta_{\operatorname{mac}}>54^{\circ}$ (not plotted). The fitted parameters based on data taken for $10^{\circ} \leq \theta_{\text {inc }} \leq 53^{\circ}$ with $\psi_{\text {pol }}=30^{\circ}, 45^{\circ}$ and $60^{\circ}$ are: $n_{1}=1.58,\left(n_{1}-n_{2}\right)=2.2 \times 10^{-5},\left(n_{1}-n_{3}\right)=5.83 \times 10^{-4}$ and $\psi_{\text {m-plene }}=1.6^{\circ}$. We also measured a point in the region with grooves. This time we found that, for $\theta_{i b c} \geq 25^{\circ}$, the measured curves cannot be matched. The measurement was repeated for the same point, but with the side of grooves facing backward, so that the grooves were not filled with the index-matching fluid. In this case there were a series of diffracted beams and we measured the one corresponding to the zeroth order pattern. The measured values for $\theta$ and $\varepsilon$ in the range of $10^{\circ} \leq \theta_{\text {inc }} \leq 60^{\circ}$ were the same as those with the filled grooves.

\section{III.5 Sample PC3.5C01 and PC3.5C02}

These two MO disks are from the same series of product with quadrilayer structure. The 
measured point was located $3 \mathrm{~cm}$ from the disk center and in the middle of a sector with grooves. The data were taken with $\Phi_{\mathrm{inc}}=90^{\circ}$ and $\psi_{\mathrm{pol}}=60^{\circ}$. The measured data and the best fit are shown in Fig. 12. The fitted parameters are: $n_{1}=1.58,\left(n_{1}-n_{2}\right)=2.3 \times 10^{-5},\left(n_{1}-n_{3}\right)$ $=5.34 \times 10^{-4}$, and $\psi_{\text {in-plune }}=-2.9^{\circ}$. The principal axes are in fact along the radial and track directions. To obtain the best fit, the quadrilayer structure must be used and adjusted in the calculation. We found that the searched structure parameters were not unique. However, the searched birefringent parameters were always the same and hence are reliable. The reason is that the measured $\theta$ and $\varepsilon$ contain information about the phase shift contributed by the birefringence, but not much information about the absolute reflectivities which are signatures of the quadrilayer. The same measurement was performed for PC3.5C02. The fitted parameters are: $n_{1}=1.58,\left(n_{1}-n_{2}\right)=2 \times 10^{-5},\left(n_{1}-n_{3}\right)=5.51 \times 10^{-4}$, and $\psi_{\text {in-pleac }}=-6.5^{\circ}$. Compared with the bare substrates PC3.5B01 and PC3.5B02, we see that the coated substrates have larger in-plane birefringence but smaller vertical birefringence. The decrease of vertical birefringence due to coating was also reported in [11].

\section{III.6 Sample PC5.25C01}

The sample is a small section of coated substrate with grooves cut from a 5.25" MO disk. The data were taken with VAE633 at $\Phi_{\text {icc }}=90^{\circ}$ for $\psi_{\text {pol }}=45^{\circ}$ and $60^{\circ}$. As shown in Fig. 13, both measured curves can be fitted very well with a fitting error of $1.65^{\circ}$, using the set of parameters: $n_{1}=1.58,\left(n_{1}-n_{2}\right)=0.9 \times 10^{-5}$, and $\left(n_{1}-n_{3}\right)=4.61 \times 10^{-4}$ and $\psi_{\text {i. -pleac }}=8^{\circ}$. 


\section{III.7 Sample PC5.25C02 and PC5.25C03}

These two samples are from the same series of product. For PC5.25C02 we measured 4 points, each located at the same radial position $(5 \mathrm{~cm}$ from the disk center) but in the middle of different sectors. $\Phi_{\mathrm{icc}}=90^{\circ}$ and $\psi_{\mathrm{pot}}=60^{\circ}$ were fixed. The measured data were nearly identical for the 4 points, see Fig. 14, indicating that the birefringence property is uniform along the track. The best match for one of the measured points (solid lines in Fig. 14) gives: $n_{1}=1.58,\left(n_{1}-n_{2}\right)=$ $0.7 \times 10^{-5}$, and $\left(n_{1}-n_{3}\right)=3.37 \times 10^{-4}$ and $\psi_{\text {in-plenc }}=-18^{\circ}$.

For PC5.25C03 we measured one point at $5 \mathrm{~cm}$ from the center of the disk and in the middle of a grooved sector. The fitted parameters are: $n_{1}=1.58,\left(n_{1}-n_{2}\right)=1.9 \times 10^{-5},\left(n_{1}-n_{3}\right)=$ $3.47 \times 10^{-4}$ and $\psi_{\mathrm{im}-\mathrm{plenc}}=11.5^{\circ}$. Figure 15 shows the measured data for both PC5.25C02 and PC5.25C03 with $\psi_{\text {pol }}=60^{\circ}$, emphasizing the similarity of the two disks.

\section{CONCLUDING REMARKS}

In this paper we have described an approach to measuring the birefringence for optical disk substrates using ellipsometry. The procedure consists of measurement of the rotation and ellipticity, and data fitting using a computer program. Using this method we have measured a number of bare and coated substrates. One of the main differences between our method and the previous ones is that we can measure the orientation of the principal axes of the index ellipsoid. Our results confirm that the disk normal direction is always a principal axis (e.g. tilted angle of less than $0.1^{\circ}$ was found for all the measured samples). However, rotation of the in-plane axes was found to occur frequently. This rotation has been mentioned previously [9], but our work 
gives the first quantitative results. Another advantage is due to the use of the glass hemisphere which makes large propagation angles. We found that the grooves in bare (even when filled with index-matching fluid) and coated substrates have different effects on the polarization state of the beam. For bare substrates, the measured data can be matched very well when the plane of incidence is perpendicular to the grooves $\left(\Phi_{\mathrm{inc}}=0^{\circ}\right)$. The opposite is true for coated substrates, i.e., $\Phi_{\mathrm{inc}}=90^{\circ}$ gives the best match. For other values of $\Phi_{\mathrm{icc}}$ the match is usually poor, especially for data measured at large $\theta_{\mathrm{imc}}$. This dependence on $\Phi_{\mathrm{icc}}$ shows that the grooves can cause a phase shift, thus producing errors in birefringence measurement. The fact that we can get good match for data taken at $\Phi_{\text {inc }}=0^{\circ}$ for bare substrates and $\Phi_{\text {inc }}=90^{\circ}$ for coated substrates may suggest that at these particular angles the grooves have no effect on the beam, and thus the measured birefringence data are more accurate.

\section{REFERENCES}

[1] D. Treves and D. S. Bloomberg, "Effect of birefringence on optical memory systems," in Optical Mass Data Storage II, R. P. Freese, A. A. Jamberdino and M. de Haan, eds. Proc. Soc. Photo-Opt. Instrum. Eng. 262-269 (1986).

[2] A. B. Marchant, "Retardation effects in magneto-optic readout," in Optical Mass Data Storage II, R. P. Freese, A. A. Jamberdino and M. de Haan, eds. Proc. Soc. Photo-Opt. Instrum. Eng. 270-276 (1986).

[3] W. A. Challener and T. A. Rinehart, "Jones matrix analysis of magneto-optical media and read-back systems," Appl. Opt. 26, 3974-3980 (1987).

[4] T. Toda, K. Shigematsu, M. Ojima and M. Yoshihiro, "Analysis of signal-to-noise ratio in 
magneto-optical disk using a polarization simulator, "Electron. Commun. Jpn. Part 2, 72, 49-57 (1989).

[5] A. Yoshizawa and N. Matsubayashi, "Analysis of optical anisotropy of PC substrate for M-O disks and its effect on CNR," in Optical Mass Data Storage II, R. P. Freese, A. A. Jamberdino and M. de Haan, eds. Proc. Soc. Photo-Opt. Instrum. Eng. 91-98 (1986).

[6] W. Siebourg, H. Schmid, F. M. Rateike, S. Abders and U. Grigo, "Birefringence -- an important property of plastic substrates for magneto-optical storage disks," Polym. Eng. Sci. 30, 11133-1139 (1990).

[7] Ivan Prikryl, "Effect of disk birefringence on a differential magneto-optic readout," Appl. Opt. 31, 1853-1862 (1992).

[8] A. Takahashi, M. Mieda, Y. Murakami, K. Ohta and H. Yamaoka, "Influence of birefringence on the signal quality of magneto-optical disks using polycarbonate substrates," Appl. Opt. 27, 2863-2866.

[9] S. Shirouzu, K. Shigematsu, S. Sakamoto, T. Nakagawa and S. Tagami, "Refractive index ellipsiods of a polycarbonate magneto-optical memory disk substrate," Jpn. J. Appl. Phys. 28, 797-800 (1989).

[10] J. E. Hayden and S. D. Jacobs, "Automated spatially scanning ellipsometer for retardation measurements of transparent materials," to appear in Appl. Opt. (1993).

[11] A. Skumanich, "Substrate birefringence in coated optical storage disks," to appear in J. Magn. Soc. Jpn. 17, S1 (1993).

[12] M. Mansuripur, "Analysis of multilayer thin-film structures containing magneto-optic and anisotropic media at oblique incidence using $2 \times 2$ matrices," J. Appl. Phys. 67, 6466-6475 
(1990).

[13] J.D. Jackson, Classical Electrodynamics, 2nd Ed., John Wiley \& Sins, New York (1975), p.281.

[14] M.V. Klein and T.E. Furtak, Optics, 2nd Ed., John Wiley \& Sons, New York, (1986), p.84.

\section{FIGURE CAPTIONS}

FIG.1 The system setup for the variational-angle ellipsometer with wavelength $\lambda=632.8 \mathrm{~nm}$ (The VAE633 system).

FIG.2 (a) The system setup for the variation-angle ellipsometer with wavelength $\lambda=780 \mathrm{~nm}$ (The VAE780 system). (b) The hemisphere setup in transmission mode. (c) The hemisphere setup in reflection mode.

FIG.3 (a) Definition of the incident polarization angle $\psi_{\text {pol }}$. (b) The polarization ellipse of the reflected beam is characterized by $\theta_{\text {vis }}$ and $\varepsilon$. The sign of $\varepsilon$ is "-" for LEP and " + " for REP. (c) and (d): Polarization state of the beam after the QWP when the fast axis is aligned with the major axis.

FIG.4 (a) The quadrilayer structure of MO disk is used in fitting the data for coated substrate. (b) The in-plane principal axes are found rotated for some substrates, but the third one is always perpendicular.

FIG.5 The best fit for glass sample. (a) The medium is forced to be isotropic. (b) Birefringence is allowed. Data were taken with VAE633. The small fluctuations appearing in the calculated curves are due to the interference of beams reflected at the front and the back surfaces of the 
sample.

FIG.6 The best fit for sample PC3.5B01 measured with (a) $\psi_{\text {pol }}=30^{\circ}$, (b) $\psi_{\text {pot }}=60^{\circ}$. The discontinuity at $\theta_{i \mathrm{ic}}=22^{\circ}$ in (a) is explained in the text. The jumps at $\theta_{\mathrm{inc}}=27^{\circ}$ and $39^{\circ}$ are not physical, since $\pm 90^{\circ}$ stand for the same polarization plane. Data were taken with VAE633. To obtain good match for bare substrates, we found the incident plane must be perpendicular to the track direction $\left(\Phi_{\mathrm{inc}}=0^{\circ}\right)$.

FIG.7 The exchange of major axis and minor axis causes a discontinuity of $90^{\circ}$ in $\theta$ when $\varepsilon=$ $45^{\circ}$.

FIG.8 Measured data and the best fit for Sample PC3.5B02. Data were measured with VAE780 in the transmission mode.

FIG.9 Measured data and the best fit for Sample APO5.25B01. Data was taken with VAE633. The fluctuations of the calculated curves are due to the interference between beams reflected from the front and back surfaces of the substrate.

Fig.10 Measured data and the best fit for Sample PC5.25B01. Data were measured with VAE780 in the transmission mode.

FIG. 11 Measured data and the best fit for Sample PC5.25B02. The measured point locates in a region without grooves. The polarization angle is (a) $\psi_{\text {pol }}=30^{\circ}$, (b) $\psi_{\text {pol }}=45^{\circ}$, (c) $\psi_{\text {pol }}=$ $60^{\circ}$. No match has been found for $\theta_{\mathrm{icc}} \geq 54^{\circ}$ (not shown).

FIG.12 Measured data and the best fit for Sample PC3.5C01. The data were measured at $\Phi_{\mathrm{inc}}$ $=90^{\circ}$ and $\psi_{\text {pol }}=60^{\circ}$ with VAE633. In contrast to bare substrate, we found that, for coated substrates, good match can only be achieved with $\Phi_{\text {inc }}=90^{\circ}$.

FIG.13 Measured data and the best fit for Sample PC5.25C01. The data were measured at $\boldsymbol{\Phi}_{\text {inc }}$ 
$=90^{\circ}$ with VAE633. (a) $\psi_{\text {pol }}=45^{\circ}$, (b) $\psi_{\text {pot }}=60^{\circ}$.

FIG. 14 Comparison of measured data for 4 points at different sectors of Sample PC5.25C02. Solid curves are for sector 1; dashed for sector 2; dotted for sector 3 and dash-dotted for sector 4. The data were taken with VAE633 at $\Phi_{\text {icc }}=90^{\circ}$ and $\psi_{\text {pol }}=60^{\circ}$. (a) Rotation angle $\theta$, (b) Ellipticity $\varepsilon$.

FIG.15 Comparison of measured data for the same position at PC5.25C02 (solid lines) and PC5.25C03 (dashed lines). The data are almost the same for $\theta_{\text {inc }} \leq 45^{\circ}$. This shows the birefringent property in the two samples is very close. 


\begin{tabular}{|c|c|c|c|c|c|c|c|c|c|c|c|c|c|}
\hline \multirow{9}{*}{ 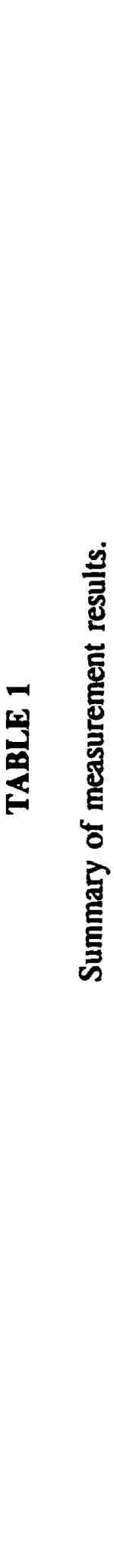 } & 总总 & $\begin{array}{l}\stackrel{0}{\circ} \\
\stackrel{0}{0}\end{array}$ & $\stackrel{n}{\check{n}}$ & $\frac{\infty}{\infty}$ & $\stackrel{\sim}{N}$ & 迨 & $\begin{array}{l}\text { ¿̊ } \\
\text { مै }\end{array}$ & $\begin{array}{l}\frac{i}{\sigma} \\
\dot{\nabla}\end{array}$ & $\begin{array}{l}\stackrel{\circ}{\sigma} \\
\sim\end{array}$ & $\frac{1}{2}$ & $\stackrel{8}{8}$ & io & రి \\
\hline & Е & $\begin{array}{l}E \\
\stackrel{E}{\Xi} \\
\end{array}$ & $\begin{array}{l}E \\
\text { E } \\
\text { 号 }\end{array}$ & 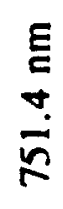 & $\begin{array}{l}\text { E } \\
\text { ○ } \\
\text { gू }\end{array}$ & $\begin{array}{l}E \\
\qquad \\
\sigma \\
\end{array}$ & 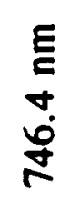 & 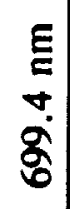 & $\begin{array}{l}E \\
\Xi \\
g \\
\dot{0} \\
\dot{J}\end{array}$ & $\frac{E}{E}$ & $\begin{array}{l}E \\
\equiv \\
0 \\
\tilde{n} \\
\tilde{n}\end{array}$ & $\begin{array}{l}E \\
\text { E } \\
o \\
\dot{\delta}\end{array}$ & 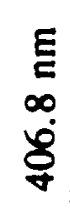 \\
\hline & $\stackrel{\overparen{E}}{\stackrel{E}{E}}$ & $\begin{array}{c}E \\
\tilde{n} \\
r\end{array}$ & $\begin{array}{l}\mathbf{E} \\
\tilde{n} \\
\end{array}$ & $\begin{array}{l}E \\
\qquad \\
\infty \\
\infty\end{array}$ & $\begin{array}{l}\underset{\infty}{E} \\
\underset{N}{N}\end{array}$ & $\begin{array}{l}E \\
E \\
\dot{+}\end{array}$ & 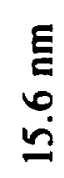 & 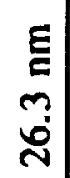 & $\begin{array}{l}E \\
\check{E} \\
\end{array}$ & $\begin{array}{l}E \\
\tilde{g} \\
\tilde{N}\end{array}$ & $\begin{array}{l}E \\
\Xi \\
\Xi \\
\Xi\end{array}$ & $\begin{array}{l}E \\
g \\
a \\
n\end{array}$ & $\begin{array}{l}E \\
E \\
\sigma \\
\dot{0}\end{array}$ \\
\hline & $\stackrel{5}{E}$ & $\frac{\dot{0}}{\frac{0}{x}}$ & $\begin{array}{l}\underset{b}{x} \\
\underset{\infty}{x} \\
\dot{\infty}\end{array}$ & 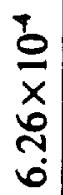 & $\begin{array}{l}\vec{b} \\
\bar{x} \\
\tilde{\infty} \\
\dot{n}\end{array}$ & $\begin{array}{l}\frac{\mathfrak{o}}{x} \\
\mathfrak{n} \\
\mathfrak{m}\end{array}$ & 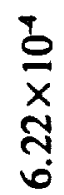 & $\begin{array}{l}\dot{b} \\
\bar{x} \\
\tilde{\infty} \\
\dot{\tilde{n}} \\
\dot{n}\end{array}$ & 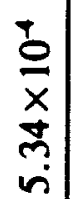 & $\frac{b}{\frac{b}{x}}$ & 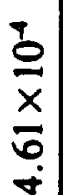 & $\begin{array}{l}\frac{b}{x} \\
\stackrel{x}{n} \\
\dot{n}\end{array}$ & $\begin{array}{l}\frac{\pi}{x} \\
\frac{\sigma}{8} \\
\dot{v}\end{array}$ \\
\hline & Ẽ & $\begin{array}{l}\stackrel{a}{0} \\
x \\
0 \\
0 \\
0\end{array}$ & $\begin{array}{l}\hat{0} \\
\tilde{x} \\
n\end{array}$ & $\begin{array}{l}\frac{n}{0} \\
x \\
n \\
0\end{array}$ & $\begin{array}{l}\hat{0} \\
\dot{x} \\
\sigma\end{array}$ & $\begin{array}{l}n \\
0 \\
x \\
\dot{y} \\
0\end{array}$ & $\stackrel{n}{\underline{x}}$ & $\begin{array}{l}\tilde{o} \\
x \\
\tilde{N} \\
\tilde{N}\end{array}$ & $\begin{array}{c}\tilde{0} \\
\bar{x} \\
\tilde{n} \\
\tilde{v}\end{array}$ & 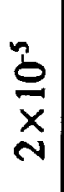 & $\begin{array}{l}\frac{n}{0} \\
x \\
a \\
0\end{array}$ & $\frac{\mathfrak{o}}{\frac{0}{x}}$ & $\begin{array}{l}\tilde{0} \\
\bar{x} \\
a \\
-\end{array}$ \\
\hline & $E$ & $\stackrel{\square}{\sim}$ & $\stackrel{\infty}{\curvearrowleft}$ & $\stackrel{\infty}{\sim}$ & $\stackrel{\infty}{\curvearrowleft}$ & $\stackrel{?}{-}$ & $\stackrel{\infty}{n}$ & $\stackrel{\infty}{\bullet}$ & $\stackrel{\infty}{n}$ & $\stackrel{\infty}{\curvearrowleft}$ & $\stackrel{\infty}{\sim}$ & $\stackrel{\infty}{\sim}$ & $\stackrel{\infty}{\curvearrowleft}$ \\
\hline & 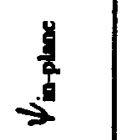 & $\begin{array}{l}\text { ஸे } \\
\dot{1}\end{array}$ & in & $\stackrel{m}{\ddot{n}}$ & $\begin{array}{l}\stackrel{0}{\circ} \\
\dot{n}\end{array}$ & $\stackrel{i}{\sim}$ & 8 & io & $\begin{array}{l}\stackrel{0}{\gamma} \\
\stackrel{\gamma}{1}\end{array}$ & $\begin{array}{l}i n \\
i p\end{array}$ & $\stackrel{\infty}{\infty}$ & $\stackrel{\infty}{\div}$ & in \\
\hline & $<$ & 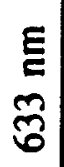 & $\underset{\tilde{E}}{E}$ & $\begin{array}{c}E \\
\tilde{\Xi}\end{array}$ & $\begin{array}{l}\text { E } \\
\stackrel{R}{R}\end{array}$ & $\begin{array}{l}\underline{E} \\
\tilde{\sigma} \\
\tilde{\sigma}\end{array}$ & $\begin{array}{l}\underline{E} \\
\stackrel{8}{\circ} \\
\stackrel{8}{R}\end{array}$ & $\begin{array}{c}E \\
\tilde{E} \\
\tilde{\sigma}\end{array}$ & $\begin{array}{c}E \\
\tilde{E} \\
\tilde{\delta}\end{array}$ & $\begin{array}{c}E \\
\hat{E} \\
\widehat{\delta}\end{array}$ & $\begin{array}{l}E \\
\tilde{\Xi} \\
\tilde{\delta}\end{array}$ & $\begin{array}{l}E \\
\tilde{\delta} \\
\tilde{\delta}\end{array}$ & $\begin{array}{c}E \\
\tilde{\sigma} \\
\tilde{\sigma}\end{array}$ \\
\hline & 号 & 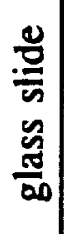 & $\begin{array}{l}\bar{\delta} \\
\tilde{n} \\
\tilde{u} \\
\tilde{\Omega}\end{array}$ & 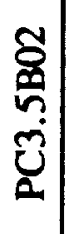 & 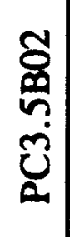 & $\begin{array}{l}\overline{8} \\
\overline{8} \\
\text { s. } \\
8 \\
\frac{8}{4}\end{array}$ & 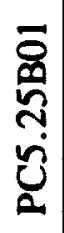 & 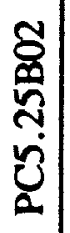 & $\begin{array}{l}\overline{0} \\
\tilde{n} \\
\tilde{u} \\
\tilde{a}\end{array}$ & $\begin{array}{l}\delta \\
\delta \\
n \\
\tilde{U} \\
\end{array}$ & 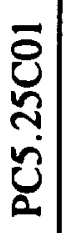 & 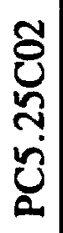 & 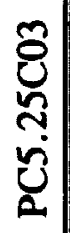 \\
\hline
\end{tabular}


The VAE633 System

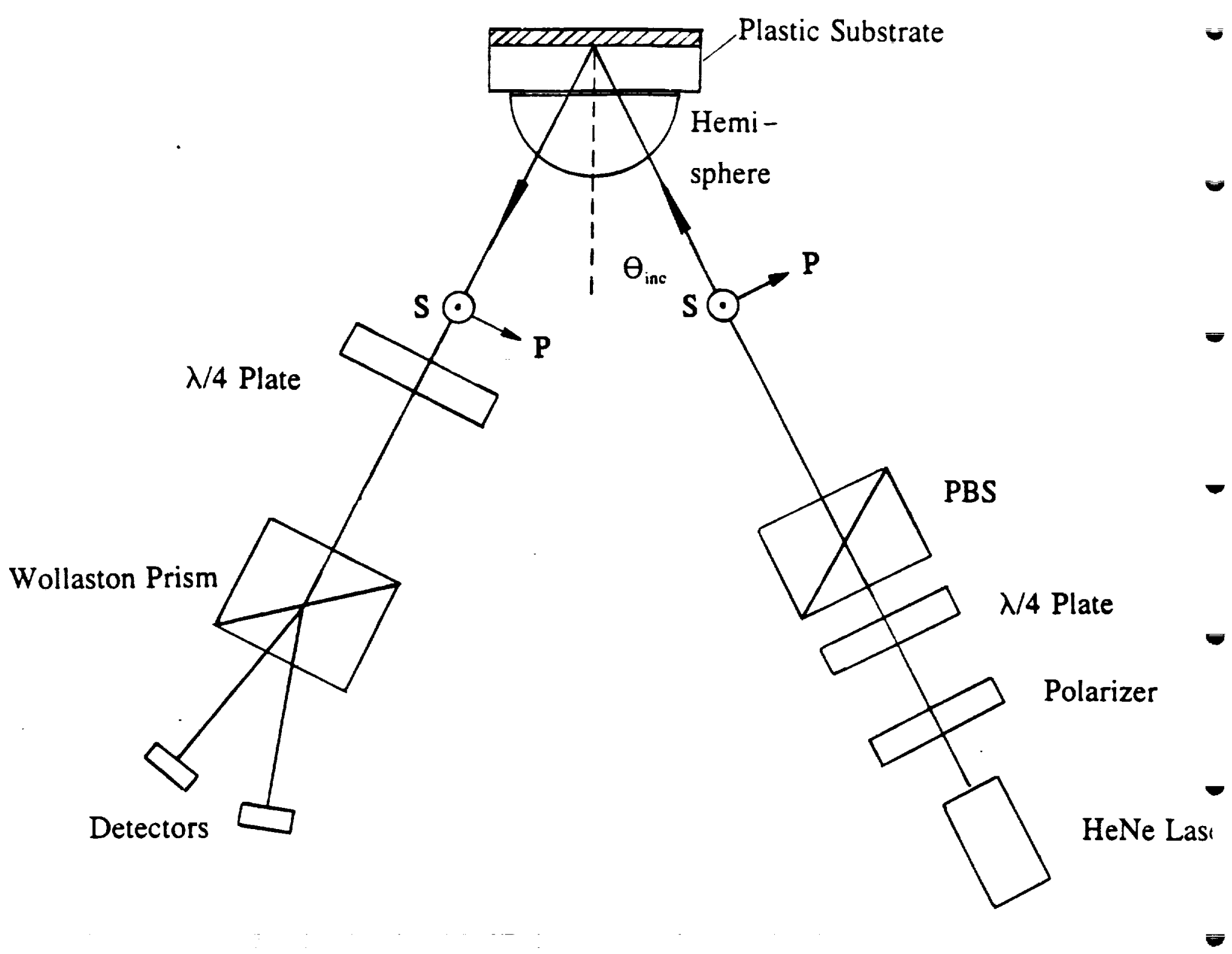

FIG.1 


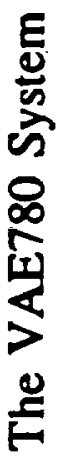
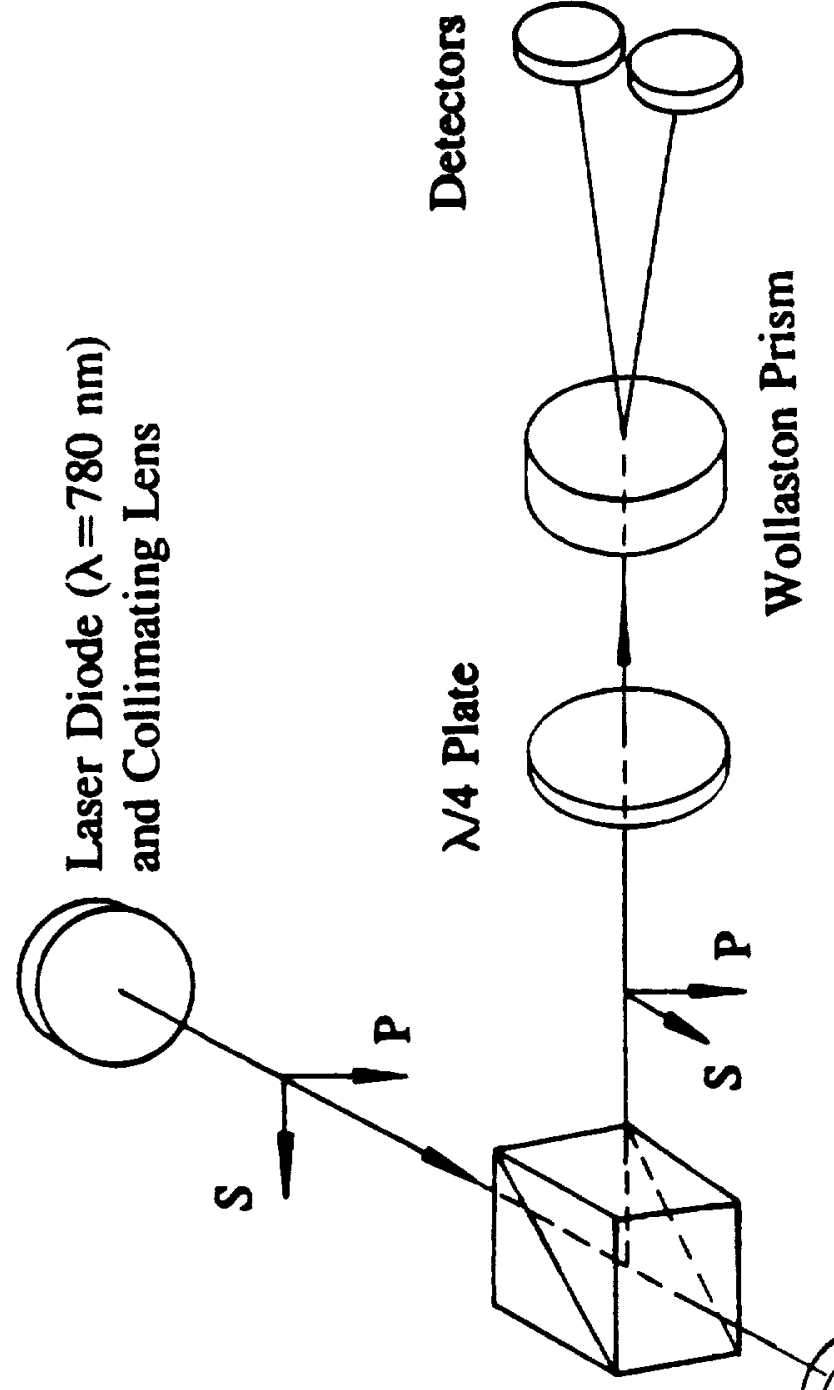

㽞

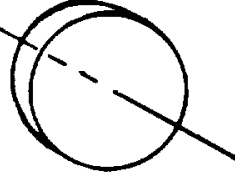

造

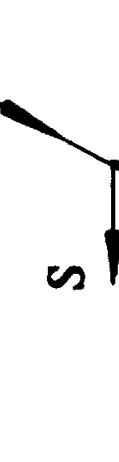




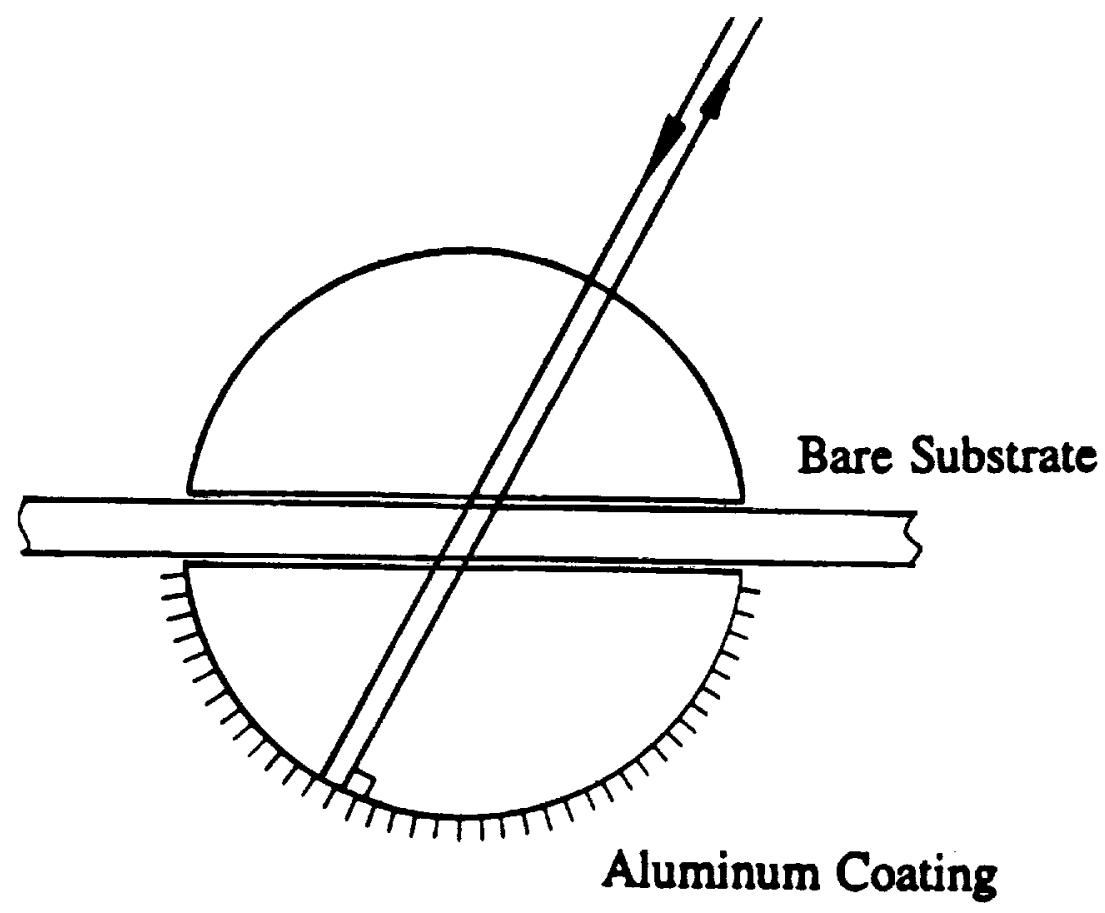

FIG.2(b)

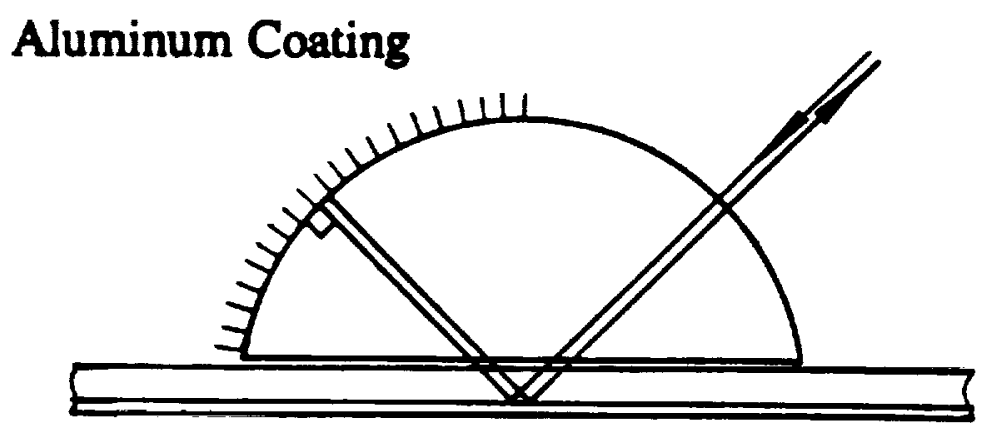

Coated Substrate

FIG.2(c) 

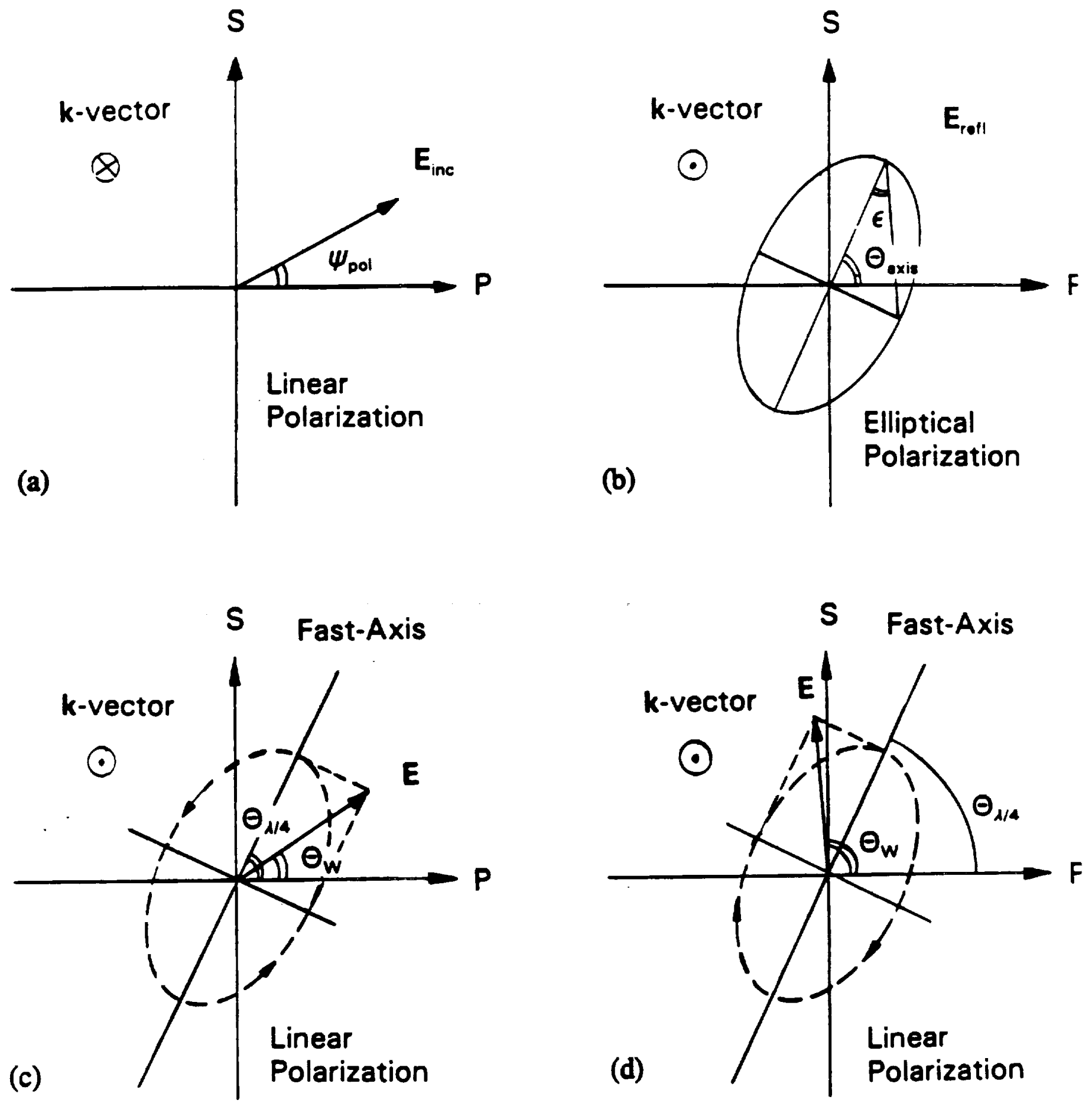

LEP: $\epsilon=\theta_{\mathrm{w}}-\theta_{\text {aris }}<0$

REP: $\epsilon=\theta_{w}-\theta_{\text {ain }}>0$ 


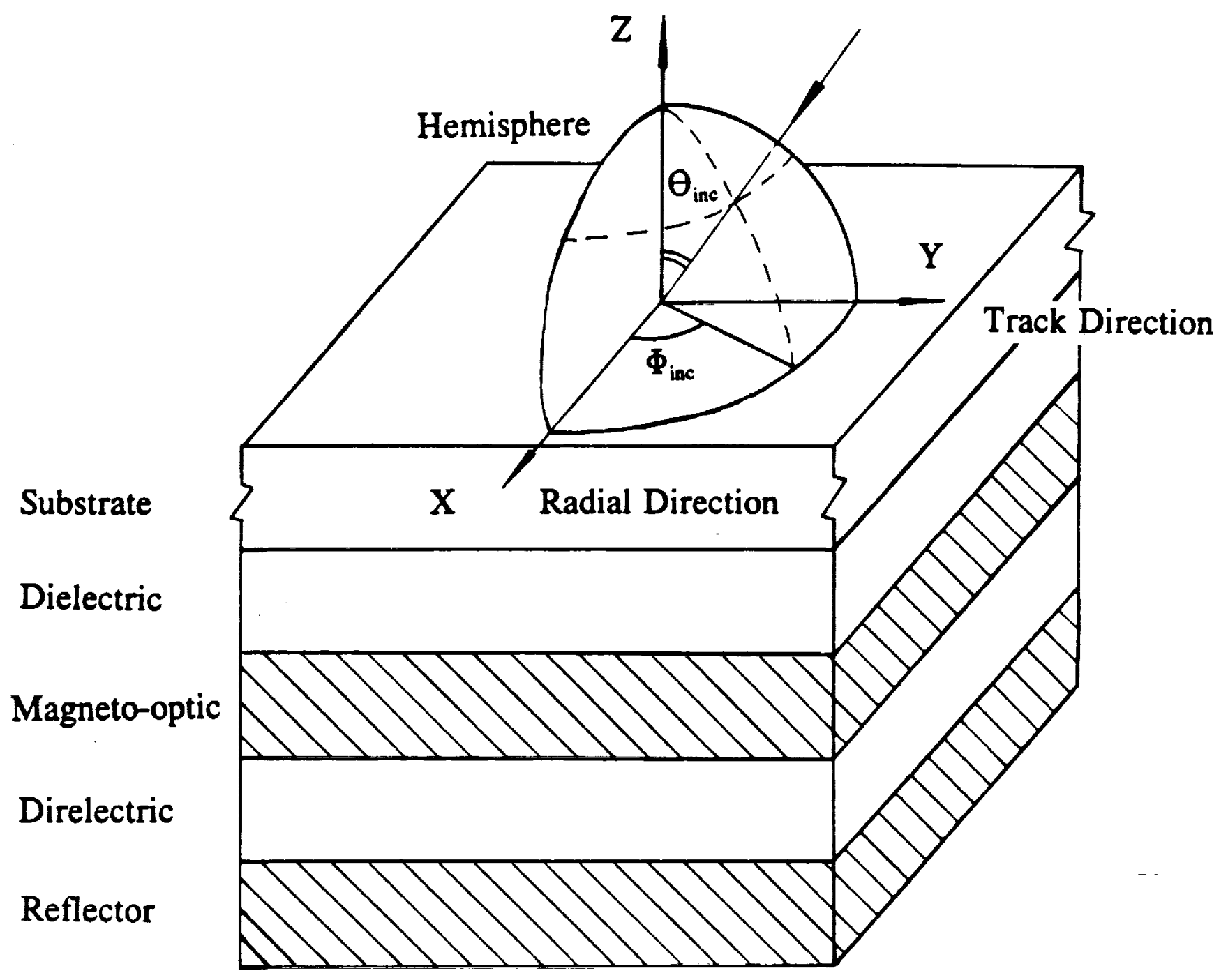

FIG.4 (a) 


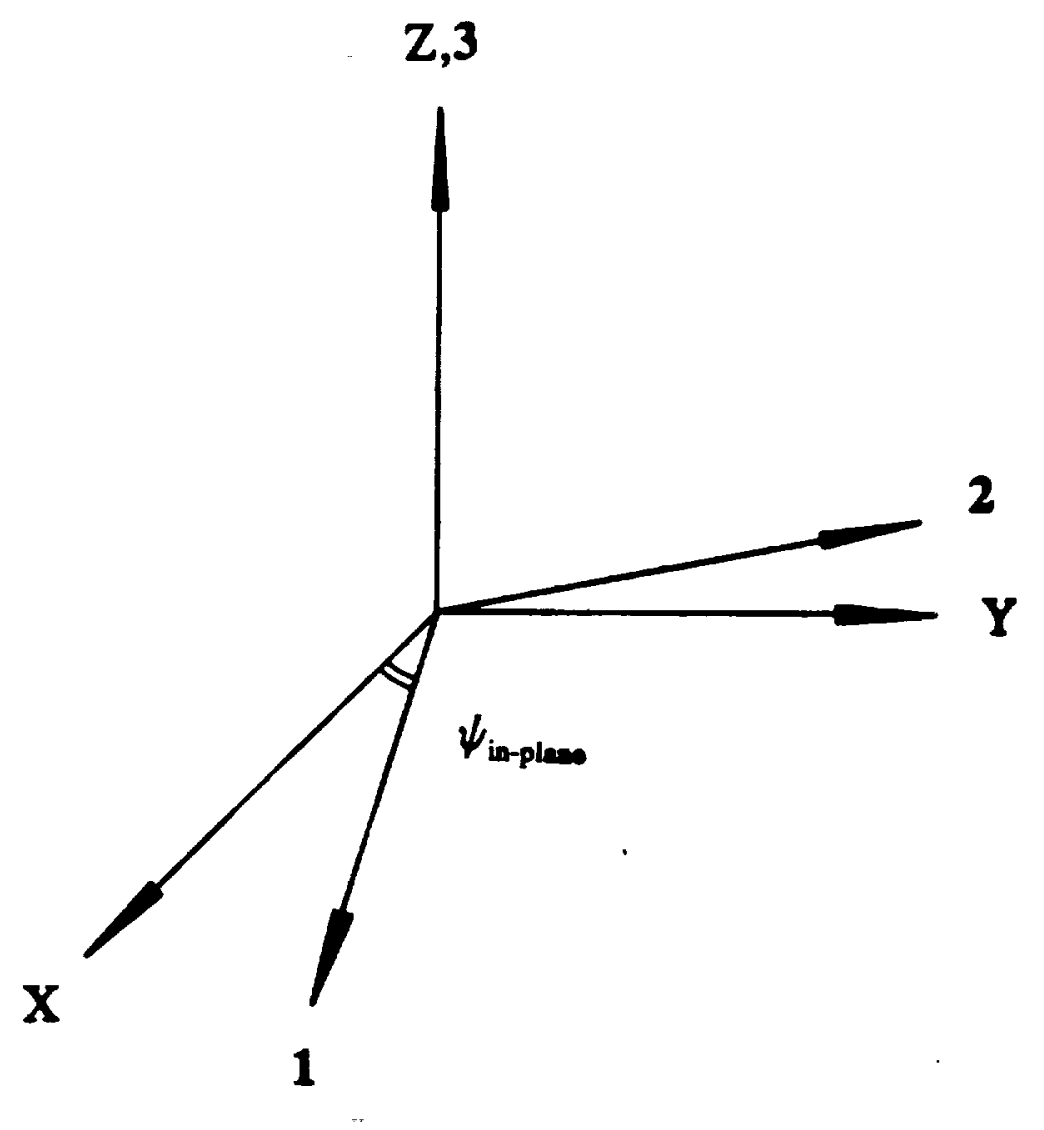

FIG.4 (b) 

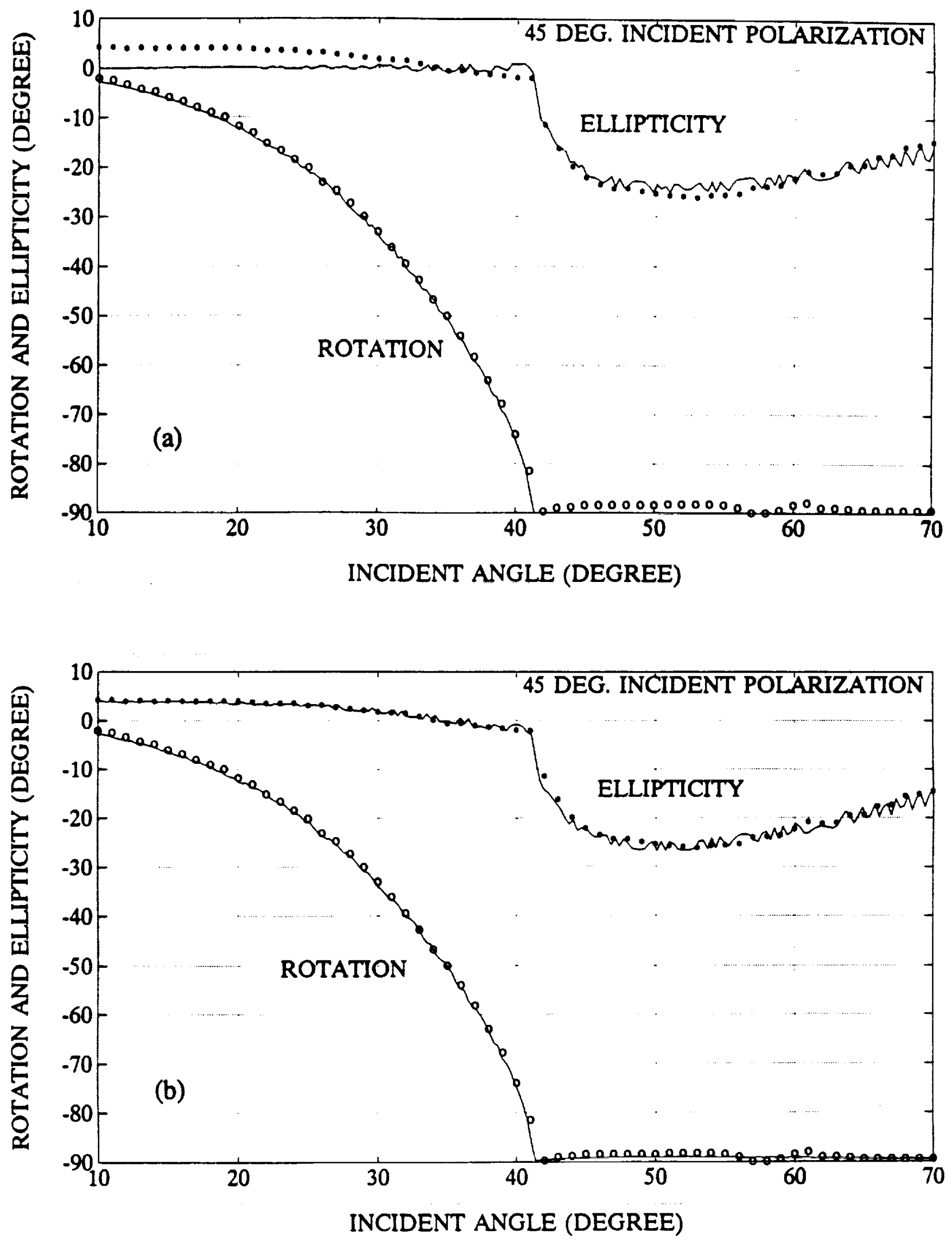

${ }_{64}$ FIG.5 

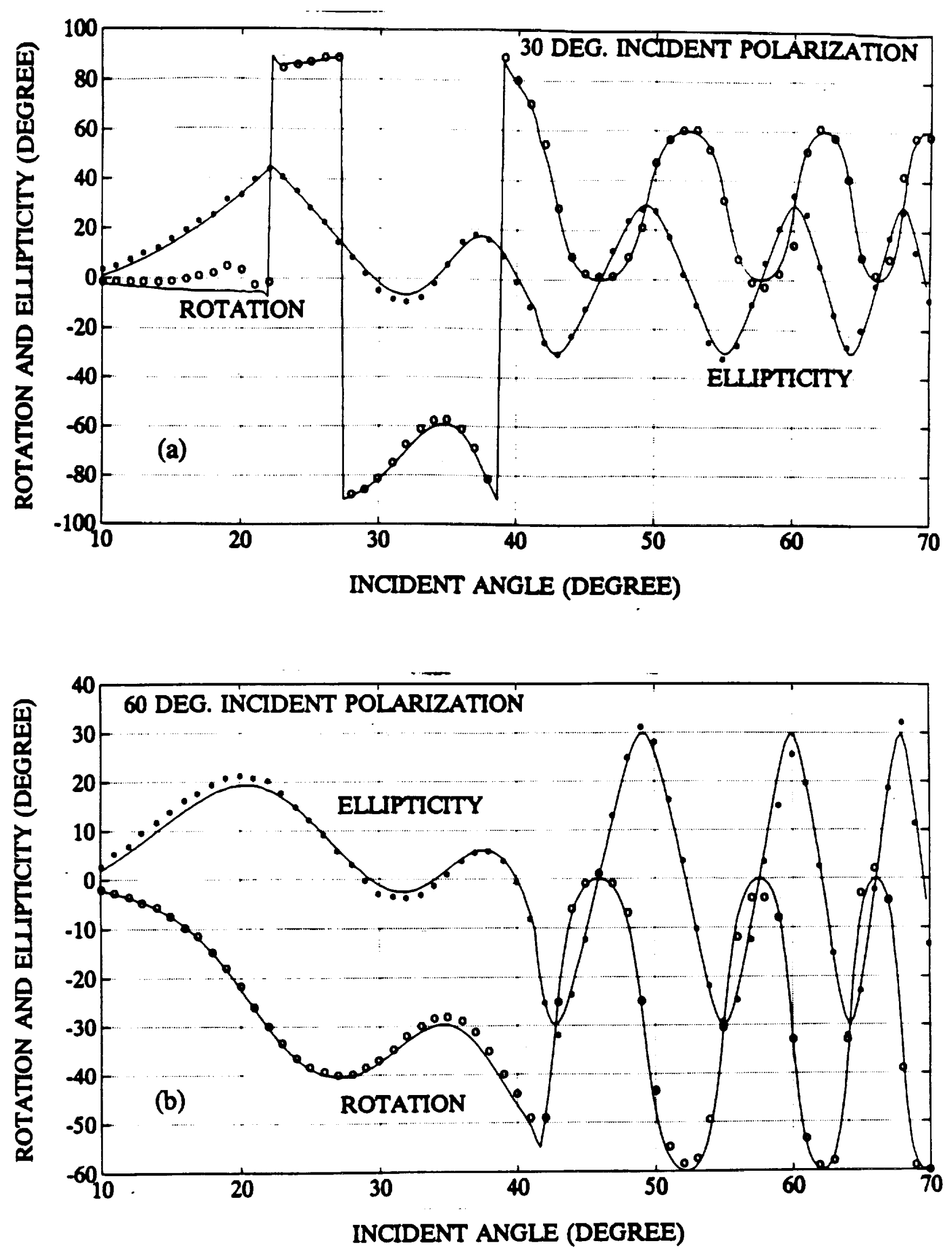

EIG.6 

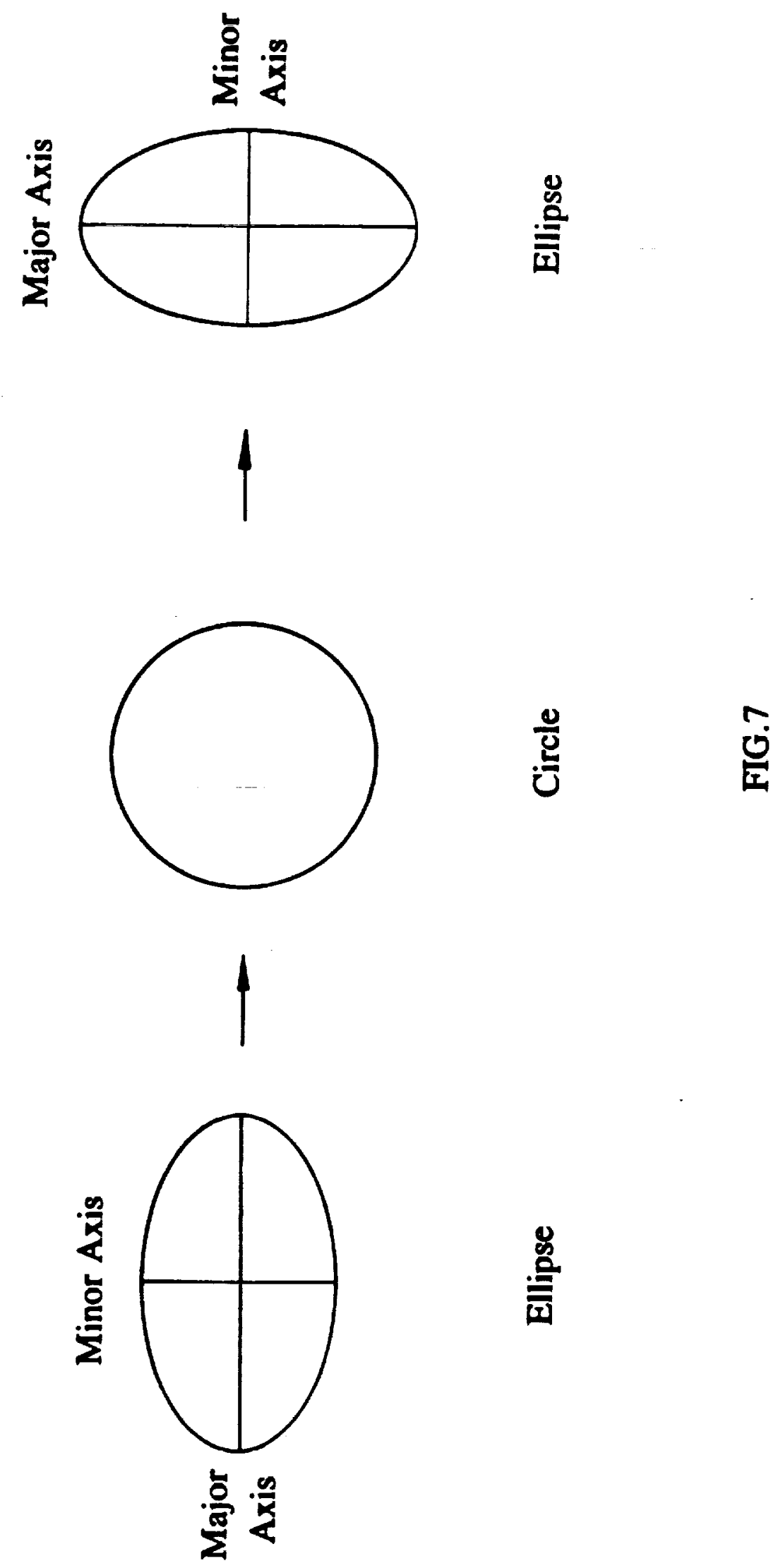

몽 


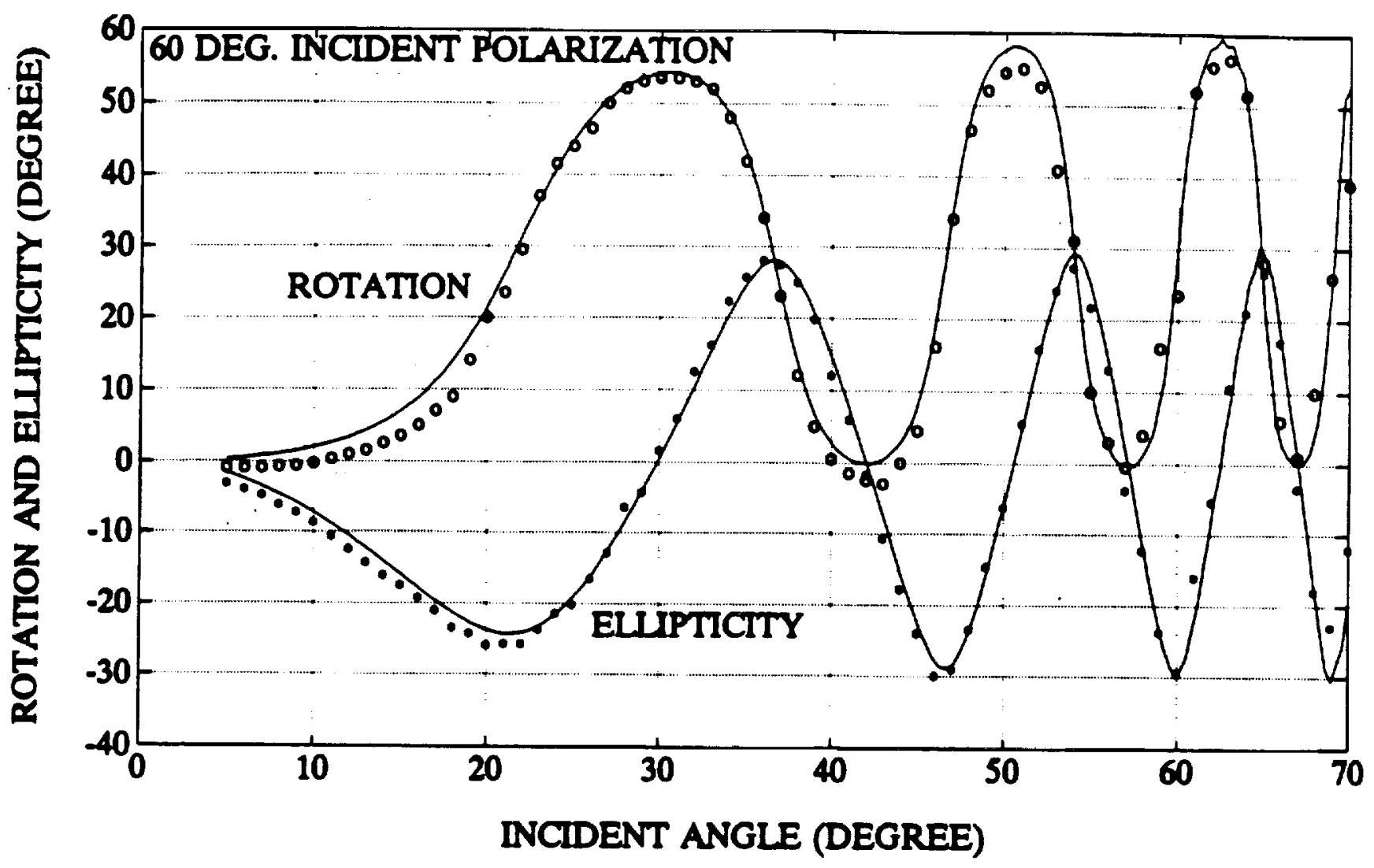

FIG.8 


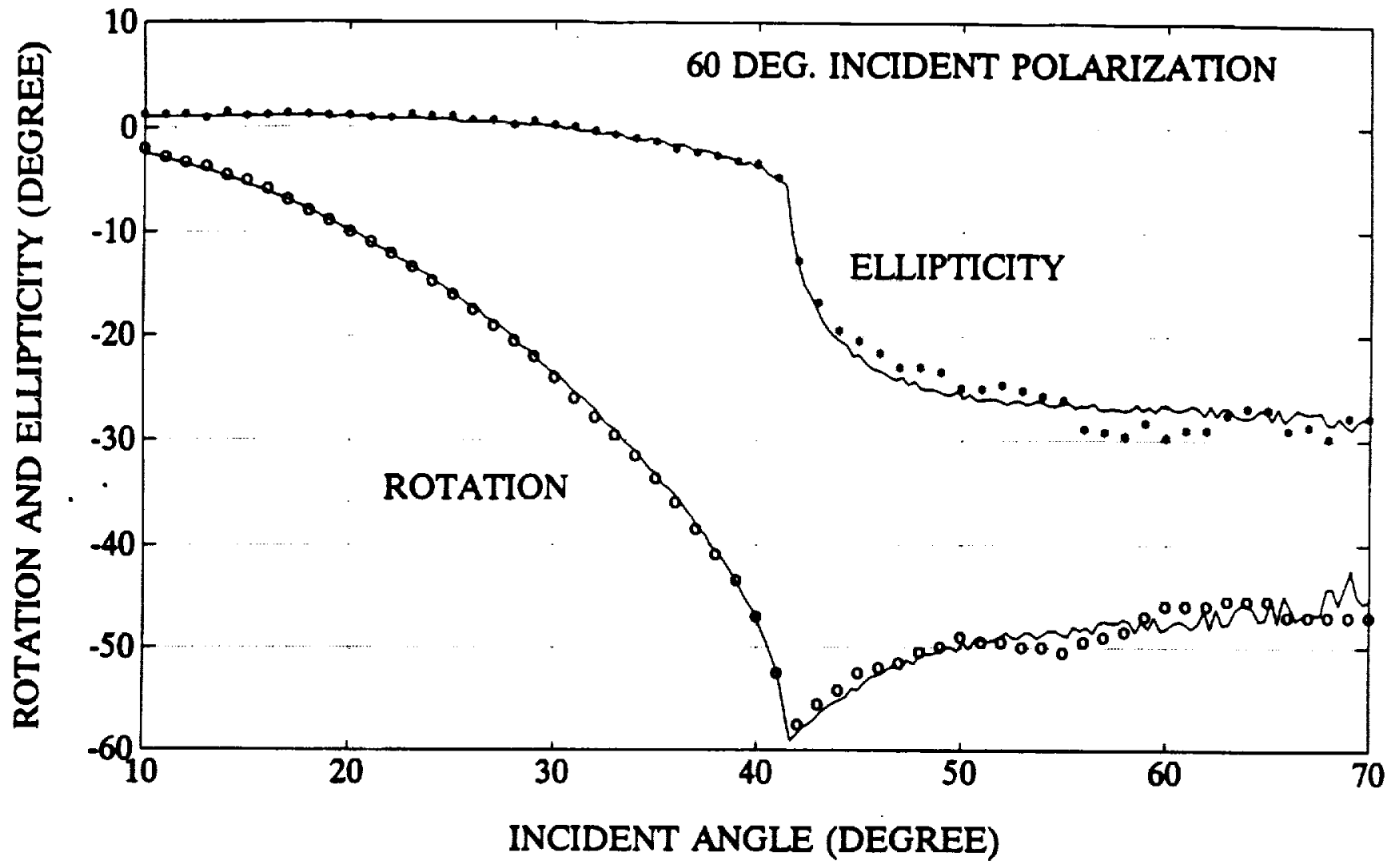

FIG.9 


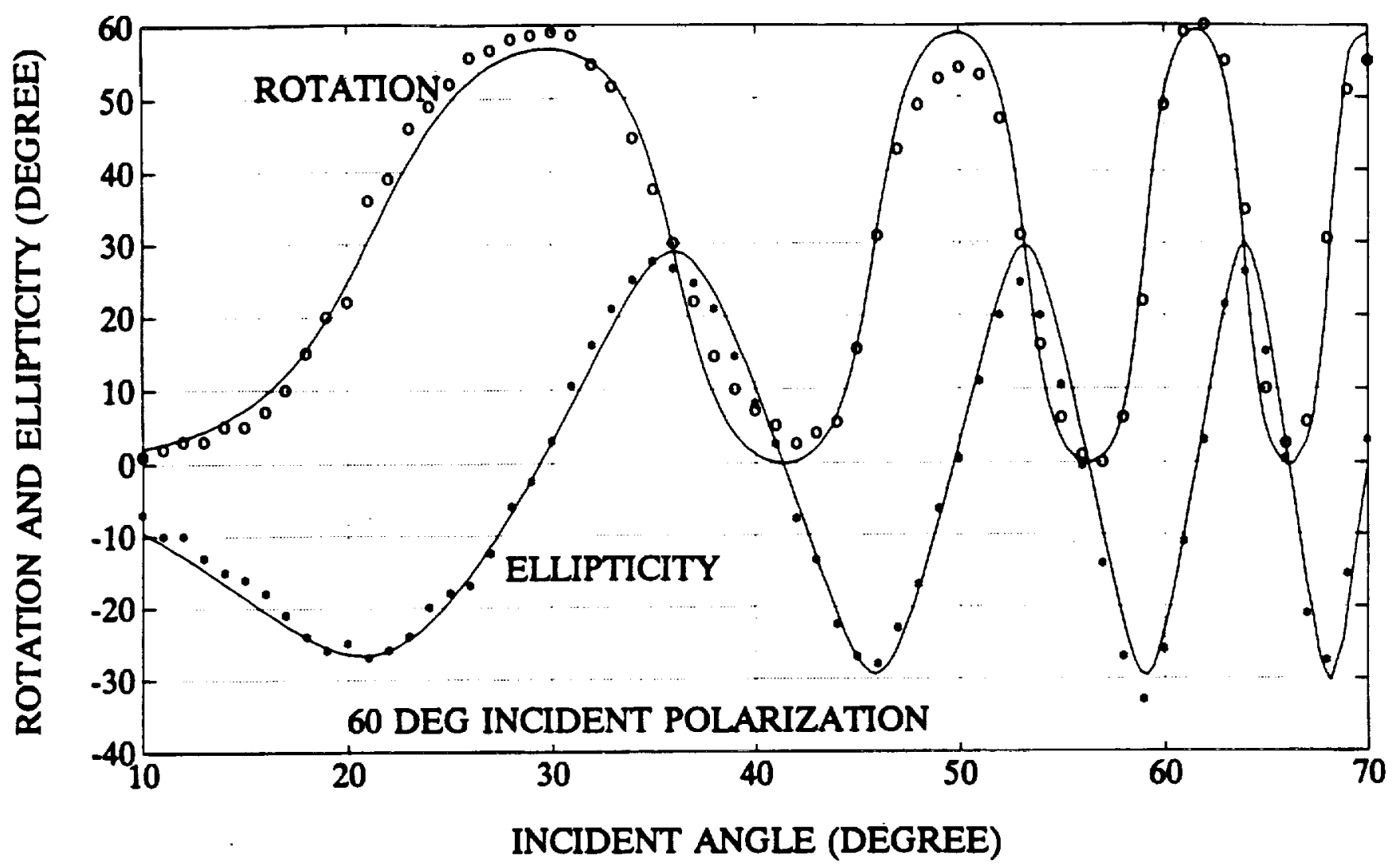

FIG.10 

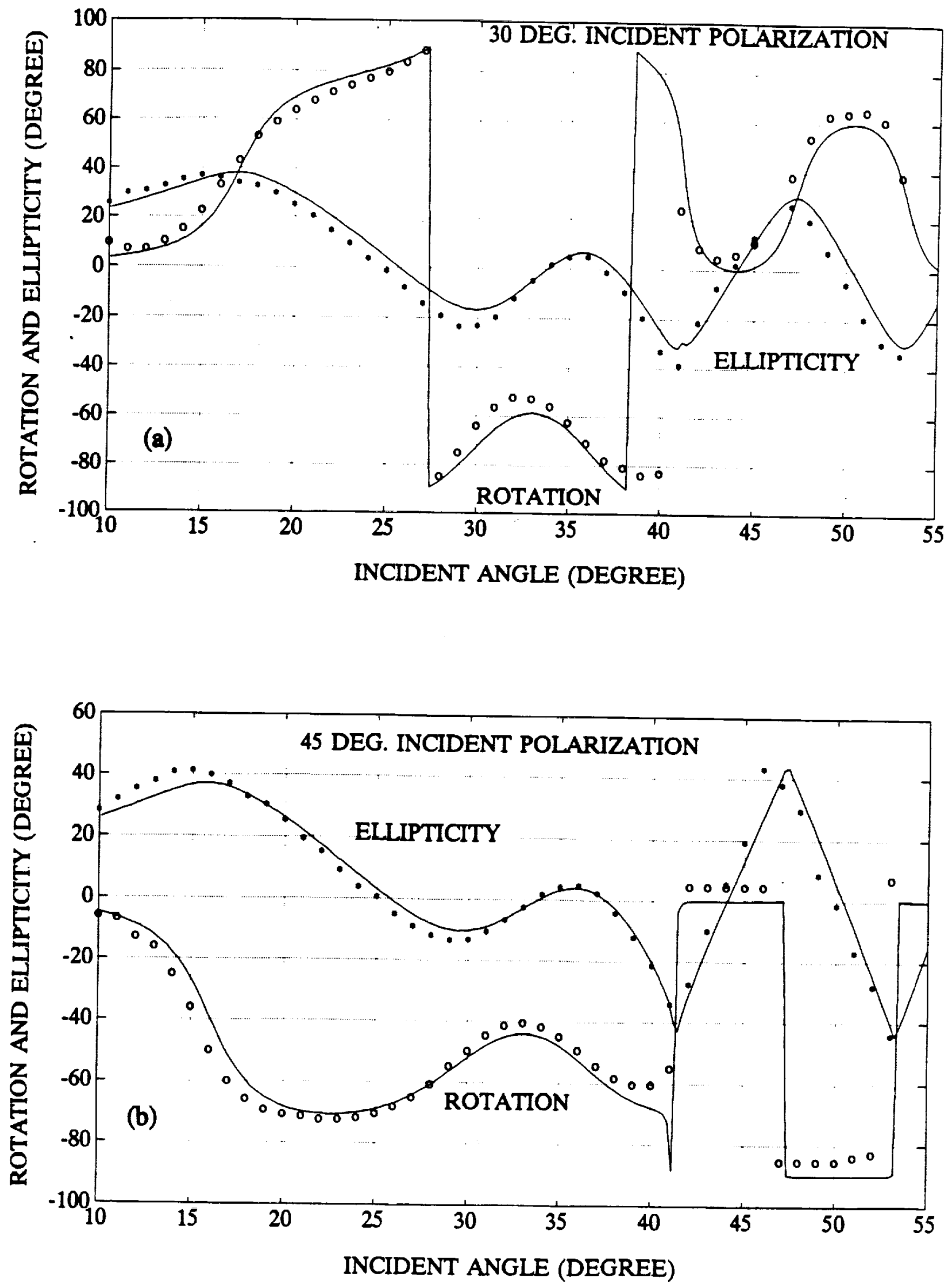

70

FIG.11 


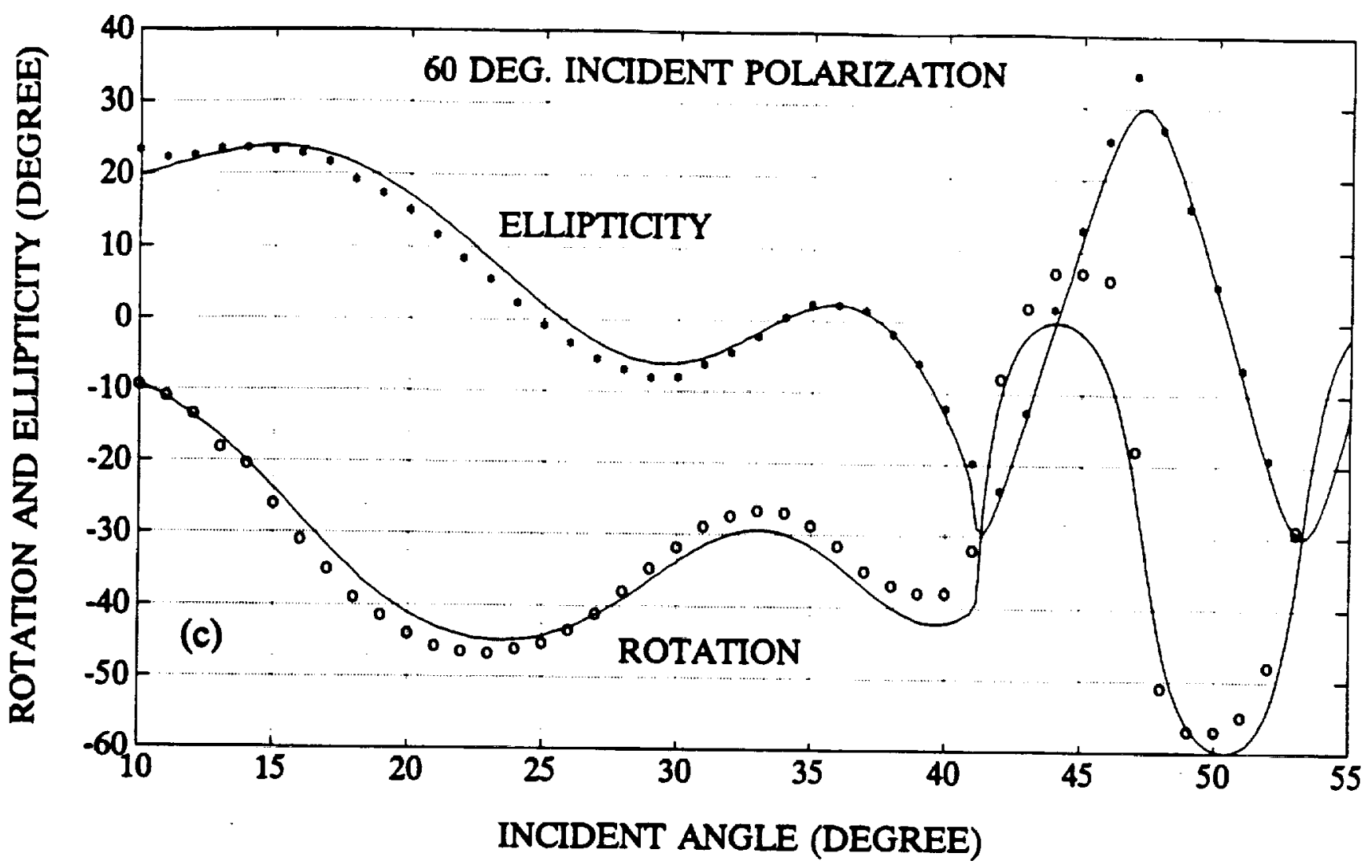

FIG.11 


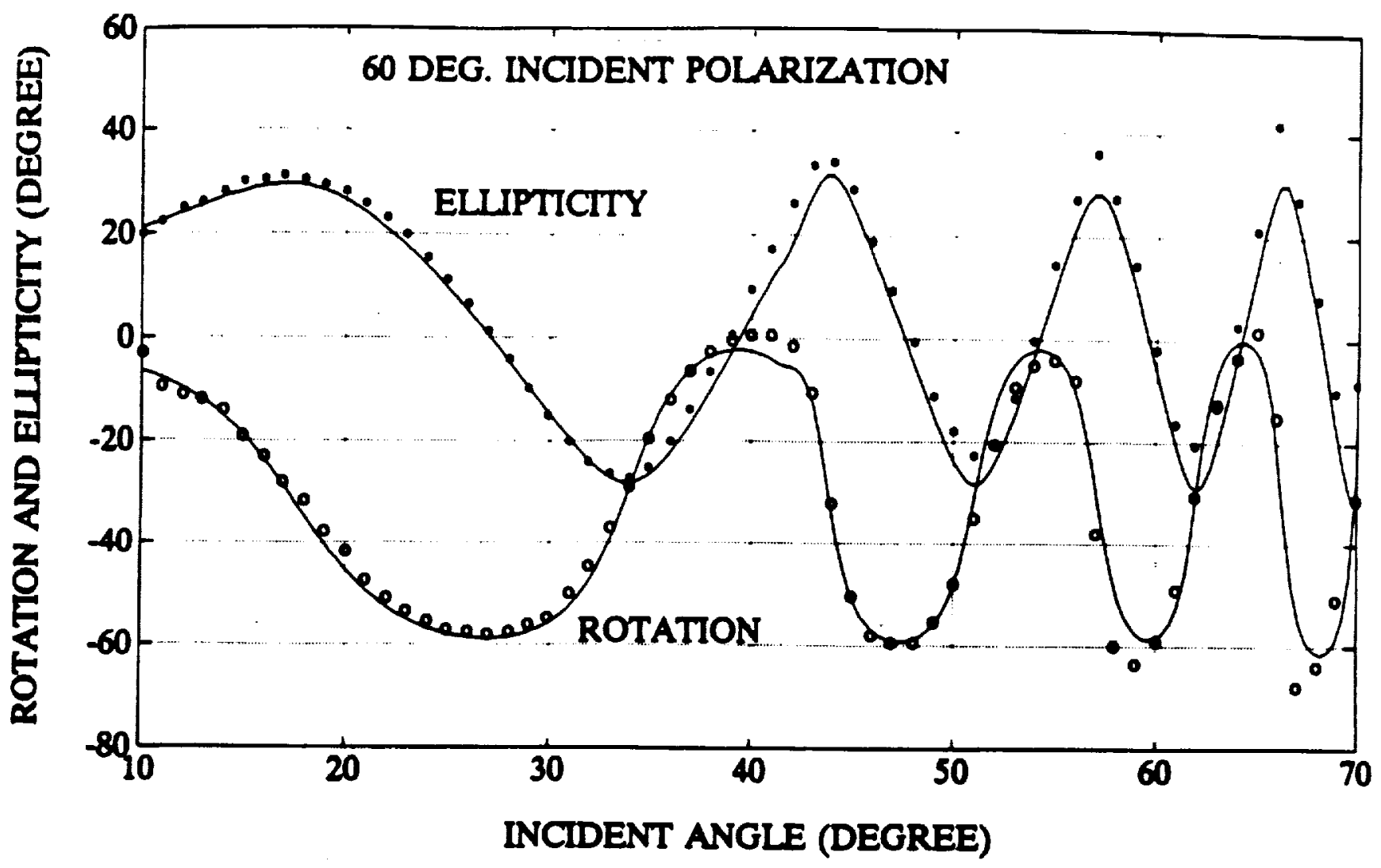

FIG.12 

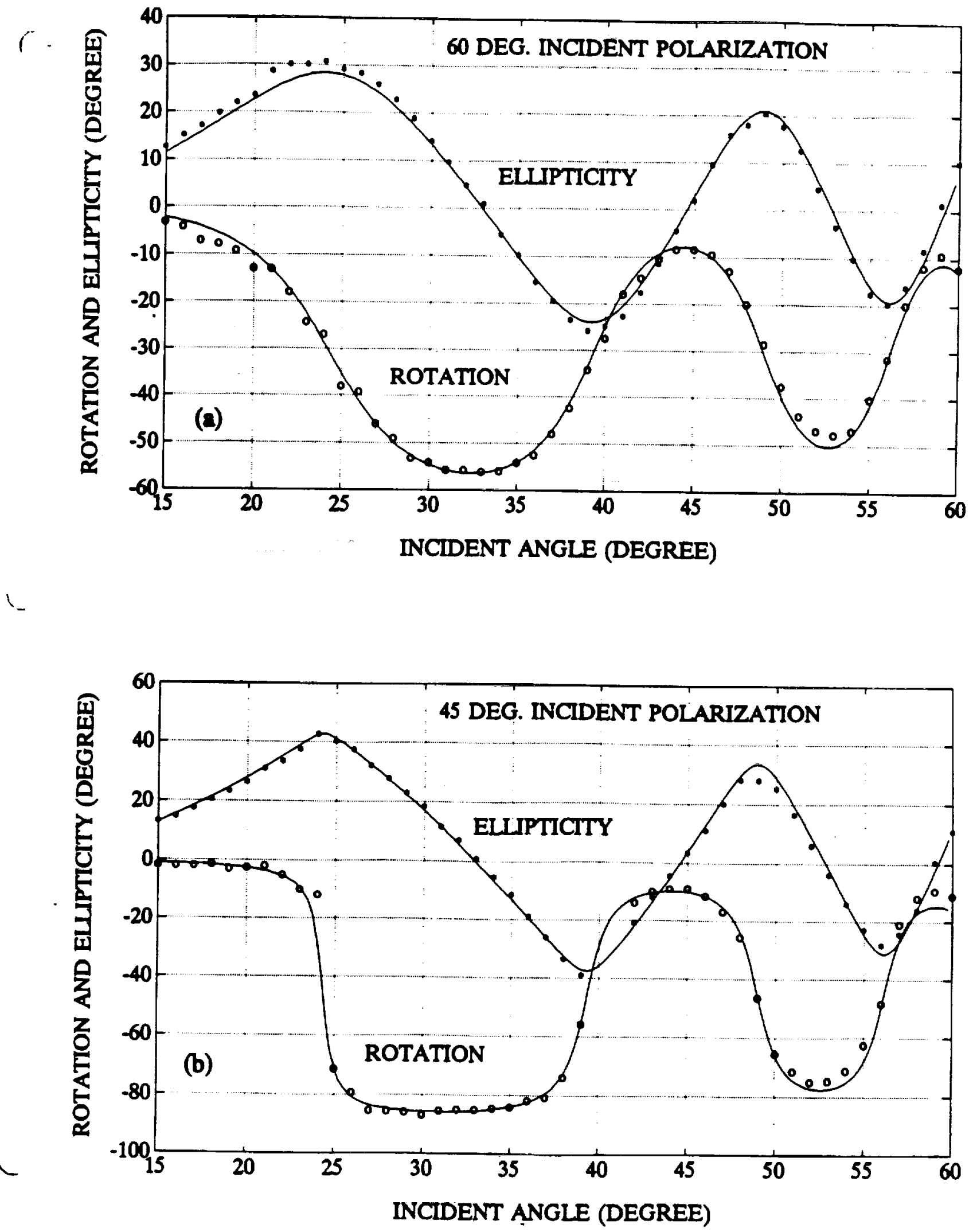

FIG.13 

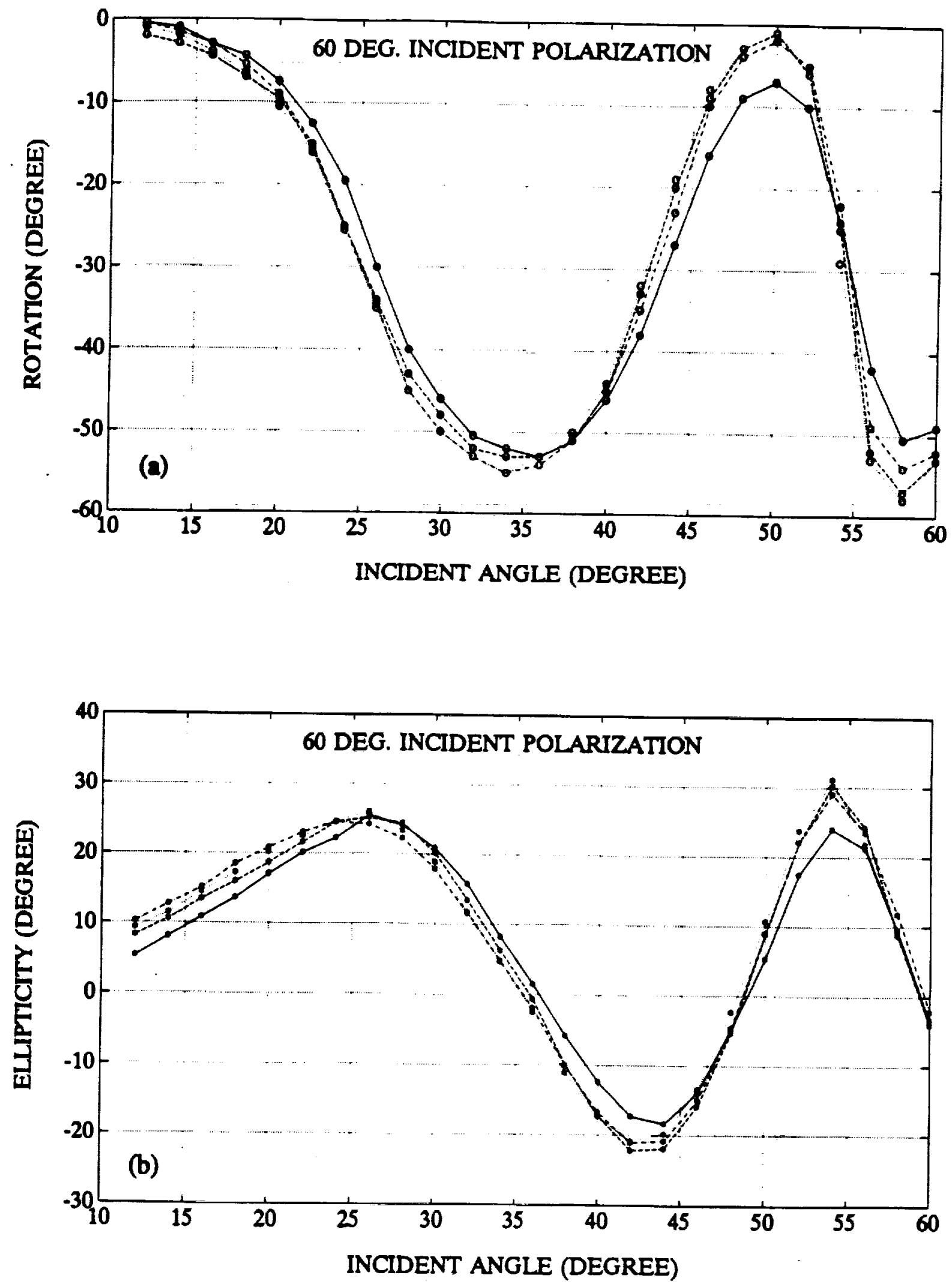

FIG.14 


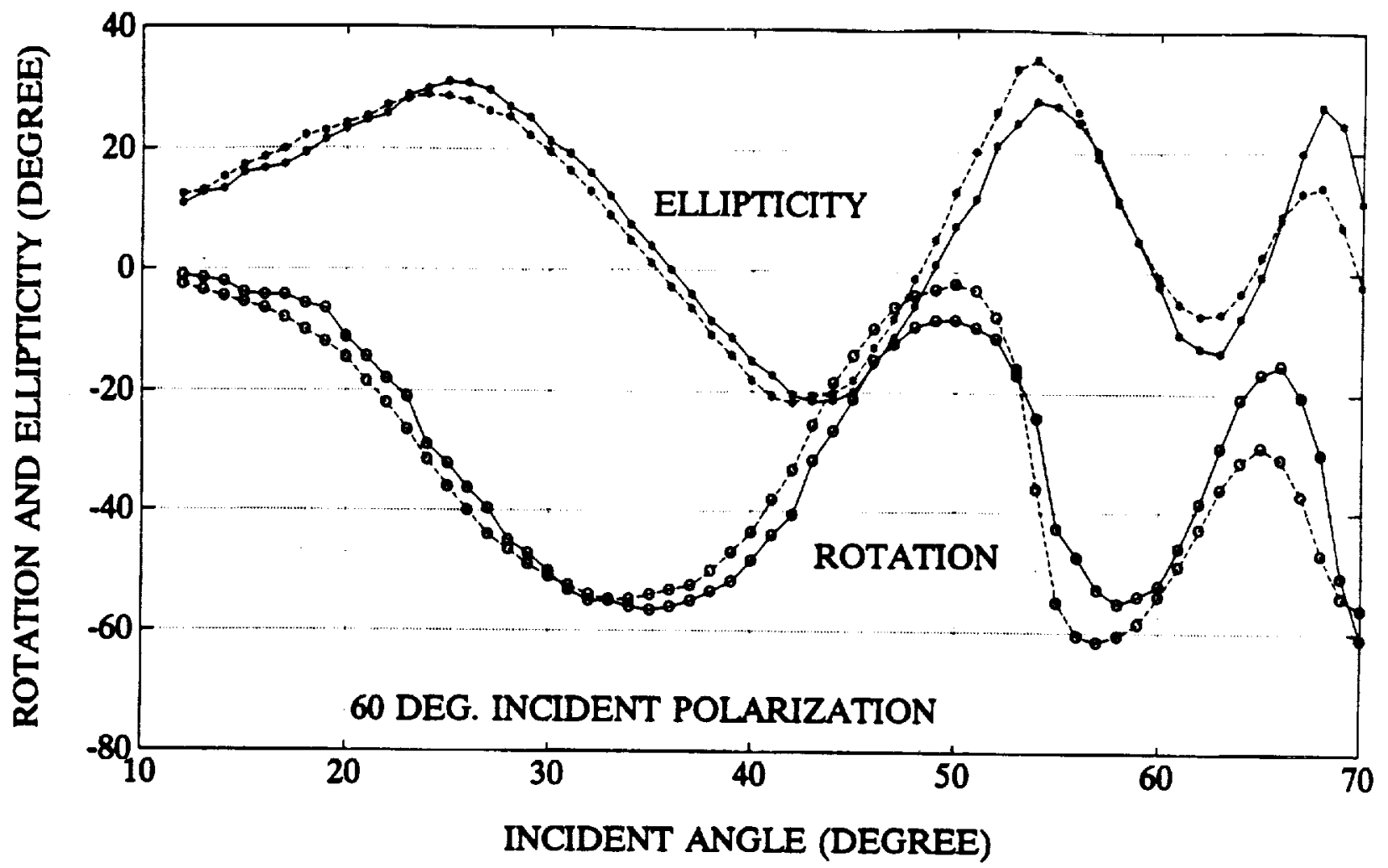

FIG.15 
-

$-$

$\bar{\sigma}$

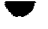




\section{APPENDIX B}

PRECEDNNG PAGE BLANK NOT FHMED

77 


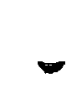

$=$

$\bar{\nabla}$

$=$ 